\title{
Soziale Herkunft, Bildung und das Alter bei der Geburt des ersten Kindes
}

\section{Johannes Huinink}

Max-Planck-Institut für Bildungsforschung Lentzeallee 94, D-1000 Berlin 33

Zusammenfassung: Die demographischen Hintergründe der Geburtenentwicklung in der Bundesrepublik Deutschland sind weitgehend aufgedeckt. Dazu gehört der Beleg erheblicher Veränderungen der kohortenspezifischen Verteilungen des Alters bei der ersten Geburt, was auf einen Wandel der zeitlichen Einpassung des Familienbildungsprozesses in die individuellen Lebensverläufe hindeuten könnte. Die soziologische Reflektion dazu steht noch weitgehend aus. Im Vergleich dreier Kohorten, 1929-31, 1939-41 und 1949-51 wird diskutiert und empirisch belegt, wie Merkmale der sozialen Herkunft und des Bildungs- bzw. Ausbildungsniveaus das Alter bei der Geburt des ersten Kindes, verstanden als Moment einer bestimmten Dimension individueller Lebensverläufe, bedingen. Auch nach Berücksichtigung des Bildungsniveaus lassen sich begründete Effekte von Merkmalen der Herkunftsfamilie identifizieren. Es zeigen sich auch deutliche Kohorteneffekte, die insbesondere in den verschiedenen Bildungs- bzw. Ausbildungsgruppen unterschiedlich ausgeprägt sind. Es kann gezeigt werden, daß die demographische Entwicklung seit den sechziger Jahren deutlich mit der Ausbildungsexpansion bei den Frauen korrespondiert, damit auf Kompositionseffekte zurückzuführen ist, aber auch mit einer zunehmenden Geburtenbeschränkung in den höheren Bildungsgruppen einhergeht.

\section{Vorbemerkung}

Das heutige, sehr niedrige Geburtenniveau in der Bundesrepublik ist in der jüngeren Geschichte entscheidend durch den rapiden Geburtenrückgang zum Ende der sechziger/Anfang der siebziger Jahre, „vorbereitet" worden. Demographisch ist dieser periodenspezifisch ausgewiesene Rückgang der Geburten weitgehend aufgedeckt ${ }^{1}$. Die soziologischen Hintergründe der Entwicklung und ihrer Folgen für die aktuelle Situation sind dagegen noch nicht hinreichend geklärt. Diese Studie soll dazu einen Beitrag leisten.

Man kann berechtigterweise fragen, ob die Beschränkung der Analyse auf diesen vergleichsweise kleinen historischen Zeitraum überhaupt zulässig ist. Die oben benannte Phase des Rückgangs der Geburtenhäufigkeiten reiht sich in eine seit dem letzten Viertel des vorigen Jahrhunderts einsetzende Entwicklung der ,säkularen Geburtenbeschränkung" ein" ${ }^{2}$. Daraus wird geschlossen, daß der neuerliche Geburtenrückgang nur ein weiterer "Schlenker" nach unten sei, er somit auch nur im Rahmen einer größeren historischen Dimension betrachtet und verstanden werden könne.

Wenn auch einiges für diese These spricht, so konnte doch bislang keine allgemein akzeptierte

\footnotetext{
'Siehe u. a. Birg et al. 1984; Huinink 1987a; Dinkel 1983.

Zum Begriff der Geburtenbeschränkung siehe Linde 1984.
}

Globaltheorie zur Geburtenentwicklung nachgewiesen werden ${ }^{3}$. Das zeigt auch die aktuelle Diskussion um den Ansatz des ,demographischen Übergangs ${ }^{\star 4}$. Eine überzeugende Kritik dazu legt zum Beispiel Freedman in seinen Überlegungen zu Theorien des Geburtenrückgangs vor (Freedman 1979). Danach beschränkt sich dieser Ansatz auf $\mathrm{zu}$ grobe und $\mathrm{zu}$ wenige Bedingungsdimensionen gesellschaftlichen Wandels. Er kann die tatsächliche, historisch und im internationalen Vergleich festzustellende Vielfalt unterschiedlicher Entwicklungslinien nicht erklären. Freedman's Kritik wendet sich implizit auch gegen das Konzept rein makro-orientierter Theorien, wie sie die Theorie des demographischen Übergangs darstellt. Er verweist auf die Vielschichtigkeit gesellschaftlicher Wandlungsprozesse, die ein komplexeres Bedin-

\footnotetext{
' Zu dieser Diskussion siehe zum Beispiel Linde 1984; Heinsohn et al. 1979; Freedman 1979; Höhn 1986. Die Möglichkeit einer solchen Globaltheorie wird mitunter auch generell bestritten. Schubnell hat diese Auffassung zum Beispiel vertreten und statt dessen einen kumulativen Katalog von Einflußßgrößen auf das ,generative" Verhalten vorgelegt (Schubnell 1972).

${ }^{4}$ Schmid geht in einer neueren Arbeit zur Bevölkerungssoziologie explizit vom Konzept des demographischen Übergangs aus (Schmid 1984). Bezeichnenderweise finden sich aber keine überzeugenden Anbindungen dieses Ansatzes an die Analyse der aktuellen Entwicklung. Zu dieser Kritik siehe auch Mackensen 1985.
} 
gungsgefüge ihrer eigenen Entwicklung darstellen, als es Makrotheorien erfassen könnten ${ }^{5}$.

Ich will hier keinen Versuch einer allgemeinen Theorie der Fertilität, die im Grunde nur ein Teil einer allgemeinen Theorie gesellschaftlichen Wandels sein kann, vorstellen oder explizit aufgreifen ${ }^{6}$. Ich werde, gleichwohl auf der Grundlage eines konzeptuellen Rahmens, der mir für eine bestimmte Ebene der Analyse gesellschaftlichen Wandels geeignet erscheint, und auf der Basis einer individuellen Lebensverlaufsanalyse einige zentrale Aspekte der Nachkriegsentwicklung bei den $\mathrm{Ge}$ burten, hier speziell den ersten Geburten, aufzeigen. Im Vordergrund stehen dabei Überlegungen zur Bedeutung der sozialen Herkunft und der Bildung für individuelle Familienbildungsprozesse, ihres Wandels und seiner aggregativen, gesellschaftlichen Folgen in der betrachteten historischen Zeit.

Der Datensatz, der den Analysen zugrunde liegt, stammt aus dem Lebensverlaufsprojekt des Sonderforschungsbereichs 3 der DFG (Mayer 1979). Seine Struktur (Kohortendesign und Lebensverlaufsdaten) läßt eine interessante Beziehung individueller Daten zu zeithistorischen Entwicklungen zu. Die Individualdaten lassen insbesondere die differentielle Sensibilität von Individuen auf sich ändernde Opportunitätsstrukturen deutlich werden, geben damit auch teilweise Auskunft über die "Motorfunktion“ von sozialstrukturellen Teilpopulationen in solchen Veränderungs- und Wandelprozessen.

\section{Zur Fragestellung}

\subsection{Demographischer Hintergrund}

Um die Fragestellung genauer begründen bzw. präzisieren zu können, gehe ich kurz auf ein paar demographische Hintergründe der aktuellen Geburtenentwicklung in der Bundesrepublik Deutschland ein.

\footnotetext{
' $\mathrm{Zu}$ einer solchen Kritik an Theorien gesellschaftlichen Wandels überhaupt siehe z. B. auch Boudon 1983.

'Natürlich muß man versuchen, die Geburtenentwicklung oder auch den Wandel der ..generativen Struktur", um einen Begriff von Mackenroth zu verwenden, als einen Teilproze $\beta$ des gesellschaftlichen Wandels zu verstehen. Damit wäre das Problem auf eine allgemeinere Ebene verlagert und in den adäquaten Zusammenhang gestellt.
}

Der entscheidende Geburtenrückgang in Deutschland hat sich schon in der Zeit von 1900 bis etwa 1925 vollzogen. Die Gesamtfruchtbarkeitsziffer ging von mehr als 5,0 auf 2,0 zurück ${ }^{7}$. Dieses Niveau wird nach dem Krieg ab Mitte der fünfziger Jahre mit dem heute oft zitierten „Geburtenberg“ wieder übertroffen. Ihm folgt der bekannte „Pillenknick" ab Mitte der sechziger Jahre.

Es ist bislang viel zu wenig beachtet worden, daß sich hinter diesen beiden periodenspezifischen Entwicklungen erhebliche Veränderungen der Altersstruktur der Frauen bei der Geburt eines Kindes von Kohorte zu Kohorte verbergen ${ }^{8}$. Der „Geburtenberg" korrespondiert mit einer sukzessiven „Vorverlagerung“ des Alters bei der Geburt eines Kindes in den Kohorten der Kriegs- und frühen Nachkriegsjahre und geringfügig mit dem gleichzeitigen Nachholen von Geburten durch Vorkriegskohorten, die sie während der Nachkriegszeit aufgeschoben hatten. Der starke Geburtenrückgang ist dagegen durch eine erneute „Rückverlagerung“ der aggregierten, kohortenspezifischen Altersmuster bei der Geburt von Kindern in den jüngeren Kohorten mitbedingt.

Das gilt ganz besonders für die Geburt der ersten Kinder. Die absolute Anzahl zweiter und weiterer Kinder hat stark abgenommen. Der Rückgang der kohortenspezifischen Geburtenhäufigkeiten für erste Kinder ist dagegen bei weitem nicht so dramatisch, wie die Zahlen zu den einzelnen Jahren suggerieren. Nach unserer Schätzung beträgt er von der Kohorte 1935 bis zur Kohorte 1952 nur etwa $10 \%$, vom Kalenderjahr 1958 bis 1983 dage-

7 Siehe zum Beispiel Marschalck 1984. Die Gesamtfruchtbarkeitsziffer (total fertility rate) ist eine demographische, kalenderjahrspezifische Maßzahl. Sie stellt die Summe der altersspezifischen Geburtenziffern eines Jahres dar. Sie könnte damit, läßt man die Sterblichkeit einmal außer Acht, als die durchschnittliche Anzahl von Kindern interpretiert werden, die ein Mitglied einer Frauenkohorte im Verlauf ihres Lebens bekommt, wenn für sie die altersspezifischen Geburtenziffern dieses Kalenderjahres zuträfen. Sie ist daher nur mit Vorsicht zu interpretieren. Bildet man diese Summe tatsächlich bezogen auf eine bestimmte Geburtsjahrgangskohorte von Frauen (cumulated fertility rate), gewinnt man einen Hinweis auf das ...Reproduktionsniveau" dieser Kohorte.

${ }^{8}$ Dieses Phänomen wird in der demographischen Literatur mit dem Begriff der demographischen Translation bezeichnet. 


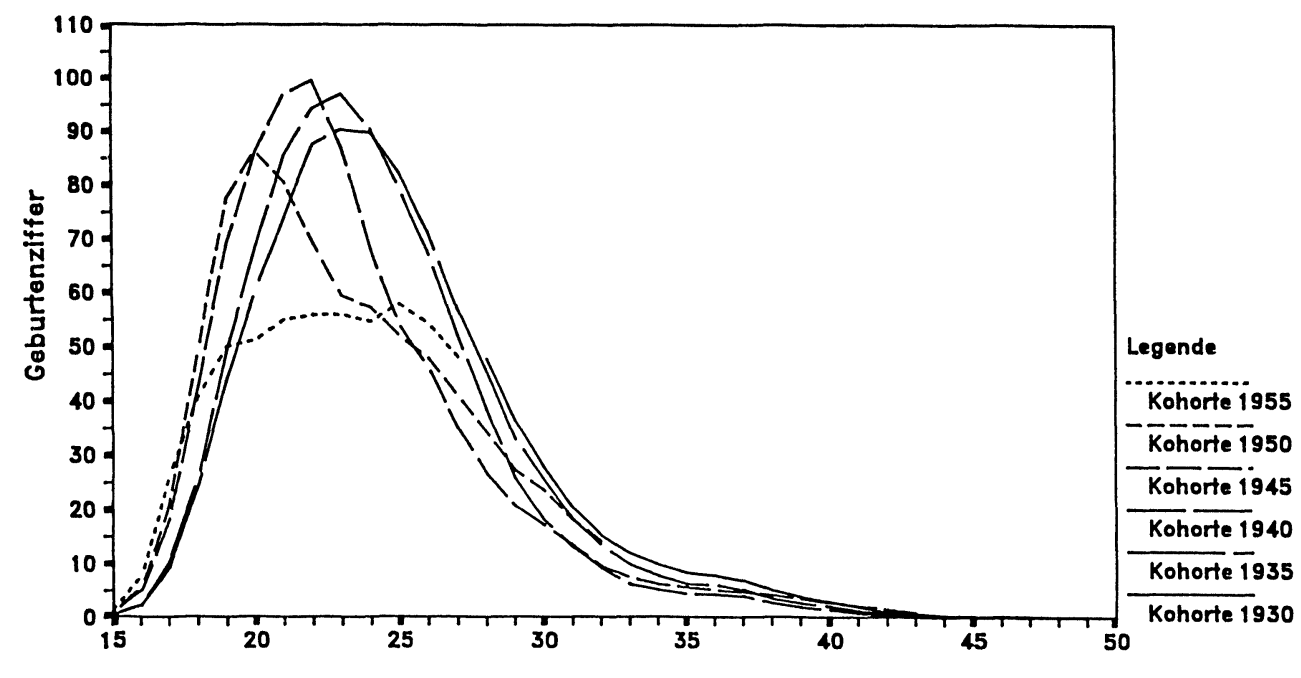

Durchschn. Alter

Graphik 1 Korrigierte altersspezifische Geburtenziffern 1. Ordnung der Kohorten 1930, 1935, 1940, 1945, 1950, 1955. (Quelle: Eigenberechnungen.)

Tabelle 1 Anteile kinderloser Frauen bzw. Männer der Kohorten 1929-31, 1939-41 und 1949-51 im Alter 22, 26,30 und 40 (in \%).

\begin{tabular}{lccccc}
\hline & & \multicolumn{5}{c}{ Alter } \\
\hline & Kohorte & 22 & 26 & 30 & 40 \\
\hline Frauen & $1929-31$ & 77.5 & 48.5 & 24.0 & 10.8 \\
& $1939-41$ & 71.3 & 32.5 & 18.1 & 11.6 \\
& $1949-51$ & 85.8 & 46.7 & 27.5 & - \\
\hline Männer & $1929-31$ & 92.4 & 65.5 & 38.4 & 12.8 \\
& $1939-41$ & 90.9 & 59.1 & 28.5 & 14.4 \\
& $1949-51$ & 91.6 & 73.3 & 50.4 & - \\
\hline
\end{tabular}

Quelle: Lebensverlaufsstudie

gen beläuft er sich auf etwa $30 \%$. Wenn man bedenkt, daß die genannten Kohorten zum großen Teil in dieser Zeit ihre Kinder bekamen, so muß man auf deutliche Effekte von Veränderungen der Altersstruktur schließen.

\footnotetext{
"Siehe Huinink 1987a. Für die anderen Ordnungen lauten die Zahlen: 2 . Ordnung: kohortenspez.: $20 \%$, periodenspez.: $36 \%, 3$. Ordnung: kohortenspez.: $49 \%$, periodenspez.: $59 \%$; 4 . Ordnung: kohortenspez.: $68 \%$. periodenspez.: $79 \%$.
}

In der Tabelle 1 sind die Anteile kinderloser Frauen bzw. Männer für die Geburtsjahrgangskohorten 1929-31, 1939-41 und 1949-51 zu unterschiedlichen Alterszeitpunkten angegeben. Sie sind auf der Basis der Daten der Lebensverlaufsstudie berechnet worden. Der Anteil Kinderloser unterscheidet sich zwischen den Kohorten in den höheren Altersstufen relativ wenig. Für die älteren Kohorten beträgt er im Alter 40 etwa $11 \%$ bzw. $13.5 \%$. Erhebliche Unterschiede zeigen sich aber im Alter 22, 26 und auch noch 30, wobei hier die jüngste Kohorte eine besondere Rolle spielt.

In der Graphik 1 sind für eine Abfolge von Kohorten die altersspezifischen Geburtenziffern für erste Geburten dargestellt ${ }^{10}$. Auch sie zeigt, daß bei der Geburt erster Kinder bedeutsame Veränderungen im Timing erfolgt sind. Dieses Ergebnis gilt natürlich zunächst nur im Aggregat; es wird zu untersu-

11) Sie sind hier als ,korrigiert" bezeichnet, da sie über cin Schätzverfahren aus geeigneten Angaben der offiziellen Statistik geschätzt worden sind. Mit den Daten des Statistischen Bundesamtes inkl. der Mikrozensen sind solche Ziffern nicht einfach zu berechnen. Das bedeutet immerhin, daß auch die Anteile kinderloser Frauen oder Männer nicht exakt zu bestimmen sind. Die Ausnahme bieten Ergebnisse der Volkszählung 1970. Siehe zu dieser Problematik Birg et al. 1984. 
chen sein, ob sich das für einzelne sozialstrukturelle Teilpopulationen bestätigen läßt.

\subsection{Untersuchungsfragen}

Ich werde versuchen, einen etwas differenzierteren Einblick in die "Anatomie" der Geburtenentwicklung nach dem Kriege aus soziologischer Sicht zu vermitteln und Bedingungs- und Prozeßzusammenhänge $z u$ identifizieren, aus denen heraus sich das skizzierte demographische Bild ergibt.

Ich beschränke mich auf erste Kinder. Die Notwendigkeit einer Differenzierung der Analyse nach der Ordnung der Geburt erschließt sich zum Teil schon aus den obigen demographischen Betrachtungen. Sie ist in soziologischen Studien zur Familienentwicklung überwiegend mißachtet worden ".

Eine explizite Einbeziehung des Eheschließungsverhaltens erfolgt nicht. Das mag auf den ersten Blick verwundern. Doch auch wenn ein gesellschaftlich immer noch stark sanktionierter, enger Zusammenhang zwischen der Geburt erster Kinder und der Eheschließung besteht, so halte ich im Rahmen dieser Analyse eine Abtrennung für vertretbar. Muß man davon ausgehen, daß beide Ereignisse gleichsam simultan eintreten, wie man vielleicht für die Zeit vor der Mitte der sechziger Jahre annehmen könnte, macht es keinen Sinn, eines der beiden Ereignisse als ..prädizierend" für das andere in das empirische Modell miteinzubeziehen. Mit der Entscheidung für eine Ehe fällt auch die Entscheidung für ein Kind. Liegen Eheschließung und Geburt des ersten Kindes zeitlich weiter auseinander, wie es seit Beginn der siebziger Jahre zunehmend zu beobachten gewesen ist, so muß angenommen werden, daß für die Geburt von Kindern Erklärungszusammenhänge virulent werden. welche die Eheschließung nicht oder noch nicht in der gleichen Weise tangieren. Das impliziert natürlich wiederum Rückschlüsse auf das vermeintlich theoretisch problemlose Verhältnis von Eheschließung und Geburt, auf die ich hier aber nicht eingehen kann ${ }^{12}$.

Eine ähnliche Argumentation wird auch zum Ereignis des Auszugs aus dem elterlichen Haushalt

"Eine weitergehende theoretische Bcgründung dieser Differenzicrung kann hier nicht ausgeführt werden.

${ }^{12}$ Kurze, eher vorläufige Uberlegungen zur differentiellen Stellung der Institutionen der Ehe und der Elternschaft im Rahmen gesellschaftlicher Wandlungsprozesse sind in Huinink 1987 a ausgeführt. greifen, das, wie im Rahmen des Lebensverlaufsprojekts nachgewiesen werden konnte, ebenfalls eng mit den Prozessen der Familienbildung verknüpft ist (Mayer/Wagner 1986).

Folgende Einzelfragen stehen daher nun im Vordergrund:

- Gibt es begründbare Unterschiede im Timing der Geburt erster Kinder, wenn man in den Kohorten nach sozialstrukturellen Gruppen mit unterschiedlicher sozialer Herkunft und Bildung differenziert?

- Wie haben sich diese gruppenspezifischen Differenzen verändert und wie sind die Veränderungen vor dem Hintergrund des gesellschaftlichen Wandels zu interpretieren?

- Gibt es weitere Ursachen für den aggregierten Wandel der kohortenspezifischen Altersmuster, wie zum Beispiel eine Veränderung der sozialstrukturellen Komposition der Kohorten, die neben möglichen durchgreifenden Verhaltensänderungen die Neuformierung von gesellschaftlichen Strukturmustern reflektieren?

Ich werde mich im folgenden vornehmlich auf die Diskussion der Ergebnisse für die Frauen konzentrieren. Ein extensiver Vergleich der beiden Geschlechter liegt außerhalb des engeren Rahmens dieses Beitrages.

\subsection{Bisherige Forschungsergebnisse}

In der soziologischen Forschung zur Geburtenentwicklung in der Bundesrepublik Deutschland haben Fragen der Dynamik der Familienentwicklung, so Analysen des Timings von Geburten, insbesondere von ersten Geburten, bislang nur wenig Beachtung gefunden. Natürlich hängt das mit der Datenlage zusammen ${ }^{13}$. Dafür notwendige Erhebungsverfahren, wie die Retrospektivbefragung oder das Mehrwellen-Panel sind in diesem Zusammenhang erst relativ spät eingesetzt worden ${ }^{14}$. Die Fragestellungen bezogen sich eher auf quantitative Aussagen zu Geburtenhäufigkeiten bzw. Kinder-

"Auf Aggregatniveau erhält man verläßliche Informationen nur bezogen auf das Timing von Geburten unterschiedlicher Ordnung innerhalb aktueller Ehen (ehedauerspezifische Geburtenziffern). Im Prinzip. aber angesichts praktischer Probleme kaum durchführbar, ließen sich auch Untersuchungen auf der Basis der Mikrozensusergebnisse denken.

" Siehe dazu die Überblicke bei Kiefl/Schmid 1985: $206 \mathrm{ff}$. 
wünschen, als auf Analysen von lebensverlaufsbzw. kohortenspezifischen Entwicklungsverläufen. $\mathrm{Zu}$ den untersuchten Bedingungsfaktoren des Geburtenverhaltens gehörte bislang nahezu immer auch das Bildungsniveau der Befragten. Es ist jedoch selten explizit auf Fragen des Timings von Geburten bezogen worden. Auch Merkmale der sozialen Herkunft sind kaum gebührend berücksichtigt worden.

Als erste demographische Studie hat wohl das Panel des Bundesinstituts für Bevölkerungsforschung Fragen des altersspezifischen Timings von Geburten miteinbezogen. Sie werden aber rein beschreibend erörtert (Pohl 1982). In diese Kategorie fällt auch die Dissertation von $\mathrm{Ch}$. Höhn zum Familienzyklus aus demographischer Sicht (Höhn 1982). Zu eher soziologisch orientierten Studien läßt sich mit Einschränkungen die Arbeit von Hoffmann-Nowotny und Höpflinger in der Schweiz rechnen (Hoffmann-Nowotny et al. 1985: $114 \mathrm{ff}$ ). Sie kommen zu dem Ergebnis, daß eine positive Korrelation zwischen dem Alter bei der Geburt und der "sozialen Schicht" (hier Ausbildung) besteht. Für die Eheschließung wird ein entsprechender Effekt nicht belegt (HoffmannNowotny et al. 1985: 131f).

Ausführlicher geht G. Scheller auf Phänomene des Wandels beim Heirats- und Familiengründungsalter ein (Scheller 1986). Sie untersucht verschiedene Heiratskohorten und kann zeigen, daß es bei den Heiratskohorten deutliche Unterschiede im durchschnittlichen Alter bei der Geburt des ersten Kindes gibt. Ihre Ergebnisse weisen auch ein mit dem Niveau bei Bildung und beruflichem Status der Befragten steigendes Alter beim ersten Kind aus. Die Ausnahme bilden die Männer ohne Beruf. Scheller konstatiert schließlich eine Zunahme des Effekts über die Ehejahrgangskohorten hin, ohne allerdings genauer die Struktur dieser Veränderung zu untersuchen. Ebenso fehlen in ihren Analysen Merkmale der Herkunftsfamilie der Befragten und multivariate Analyseansätze.

Aus dem Kontext des Lebensverlaufsprojekts am Max-Planck-Institut für Bildungsforschung liegen Arbeiten zum Ausscheiden aus dem elterlichen Haushalt und zu Eheschließungen vor. die im engeren Sinne mit der hier behandelten Problematik zu tun haben (Mayer/Wagner 1986; Papastefanou 1986a; 1986b). Darin haben EreignisdatenanalyseModelle unterschiedlicher Provenienz Anwendung gefunden.

Der Zusammenhang zwischen Merkmalen der sozialen Herkunft und des Bildungs- bzw. Ausbil- dungsniveaus mit dem Timing des Eintritts in die Elternschaft ist ausgiebiger in der amerikanischen Literatur diskutiert und mit unterschiedlichen $\mathrm{Me}$ thoden empirisch untersucht worden. Dazu gehören wiederum Verfahren der Ereignisdatenanalyse, so z.B. bei Michael/Tuma 1985, Newman/ McCulloch 1984 oder Bloom/Trussel 1984. In den beiden letzteren Arbeiten wird explizit versucht, über verschiedene Strategien einer Parametrisierung der altersspezifischen Raten des Eintritts in die Elternschaft den Aspekt des Timings der Geburt des ersten Kindes modellmäßig umzusetzen.

Rindfuss et al. 1984 z. B. schätzen Logit-Modelle bedingter Übergangswahrscheinlichkeiten zum ersten Kind für unterschiedliche Kohorten zu bestimmten Altersstufen. Man findet weiter Regressions- bzw. Strukturgleichungsmodellanalysen mit dem Alter bei der Geburt des ersten Kindes als abhängigem Merkmal, so bei Rindfuss et al. 1980, Rindfuss/John 1983 und Marini 1978, 1984, 1985. Hier wird versucht, eine interdependente Beziehung zwischen dem Bildungsniveau und dem Alter bei erstem Kind nachzuweisen ${ }^{15}$.

Die inhaltlichen Ergebnisse der oben genannten Studien decken sich mit den schon erwähnten, was die Bedeutung des Bildungsniveaus betrifft. In ähnlicher Weise wird aber auch die Relevanz von Merkmalen der Herkunftsfamilie nachgewiesen: je geringer der soziale Status der Herkunftsfamilie der Befragten ist, bzw. je größer die Herkunftsfamilie ist, um so früher bekommen die Befragten ihr erstes Kind. Gleiches gilt, wenn sie im Jugendalter nur mit einem Elternteil aufgewachsen sind bzw. wenn sie auf dem Lande wohnen. Der Ein$\mathrm{flu}$ ß der Religion $\mathrm{z}$. B. ist dagegen zunehmend $\mathrm{zu}$ vernachlässigen.

\section{Theoretische Vorüberlegungen}

\subsection{Konzeptueller Rahmen}

Den Rahmen für die folgenden inhaltlich-theoretischen Überlegungen soll ein kleines Modell liefern, das man als einen Ausschnitt eines mehrebenentheoretischen Konzepts verstehen kann. Es ist selbst als ein Teilmodell im Rahmen eines Ansatzes gesellschaftlichen Wandels einzubetten und

15 Ich lasse diesen Aspekt hier noch außer Acht, wenngleich mit den Lebensverlaufsdaten eine adäquatere Analyse dieser Beziehung unter der expliziten Einbeziehung der zeitlichen Dynamik möglich wäre. als es nichtrekursive Querschnitt-Modelle erlauben. 


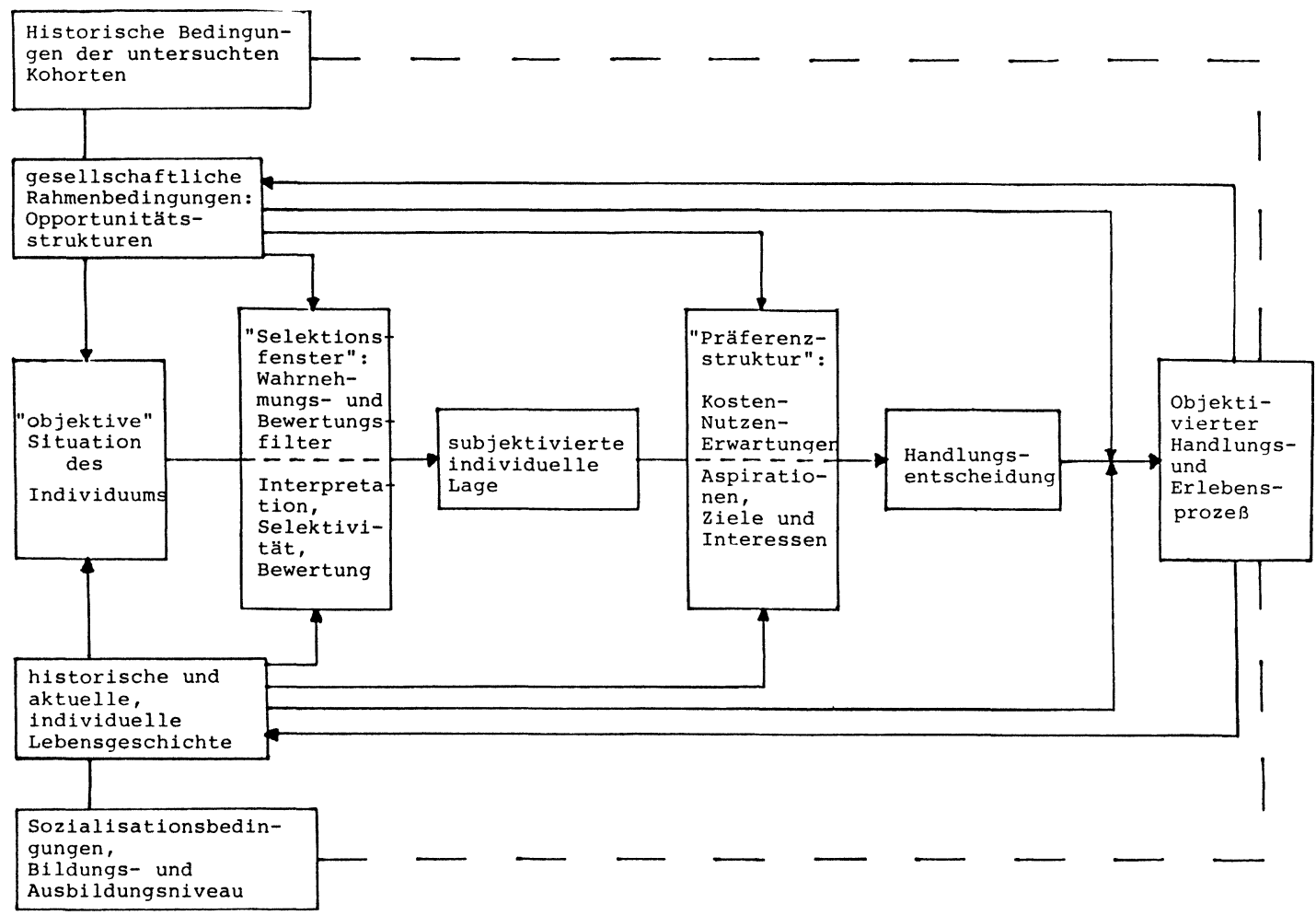

\section{Diagramm 1}

konzentriert die Aufmerksamkeit eher auf die individuelle Dimension des Prozesses.

Das Modell soll zum einen die inhaltlich-theoretische Argumentation in den Einzelthesen steuern oder kontrollieren. Zum anderen soll es zeigen, wo die empirisch nachgewiesenen Zusammenhänge in einem möglichen Erklärungszusammenhang anzusiedeln sein werden.

Als graphische Veranschaulichung dient das Diagramm 1. Die mittlere Achse des Diagramms beschreibt ein Modell eines individuellen Entscheidungs- und Handlungsprozesses: von der „objektiven" individuellen Situation über den Wahrnehmungs- und Bewertungsfilter zur subjektivierten Interpretation der individuellen Lage, über die individuelle Präferenzstruktur mit ihren Entscheidungsparametern (Kosten-Nutzen-Erwartungen und Aspirationen) zu einer Handlungsentscheidung, die in den individuellen Handlungsproze $\beta$ mündet. Er wirkt zurück auf die Bedingungen der „objektiven" Situation: die gesellschaftlichen (und netzwerkspezifischen) Rahmenbedingungen und die kumulierte individuelle Lebensgeschichte.
Sie sind in unterschiedlicher Weise mit den Prozessen der mittleren Achse zurückgekoppelt. Sie bestimmen nicht nur die „objektive" Situation des einzelnen neu, sondern bedingen auch das „Selektionsfenster" sowie die Parameter des Entscheidungskalküls (Kontrolle und Präformierung) und die Realisationschancen bzw. den tatsächlichen Effekt der Handlungsentscheidung. Der Handlungszusammenhang ist in Abhängigkeit des individuellen Kontrollbewußtseins bzw. -potentials auch immer ein Erlebenszusammenhang, innerhalb dessen dann u. a. das Auftreten bzw. Nichtauftreten der Geburt eines ersten Kindes gehört.

Die Rolle der individuellen Lebensgeschichte (Sozialisationserfahrungen. Bildung, Informationsniveau $u$.a.) trägt wesentlich zur Selbstreferentialität des Prozesses bei. Die gesellschaftlichen Rahmenbedingungen sind ebenfalls interdependent eingebunden. Auf die spezifischen Beziehungen innerhalb dieser charakteristischen Rückkopplungsstruktur gehe ich hier nicht weiter ein. Insbesondere ist der direkte Schluß vom einzelnen Individuum zu gesamtgesellschaftlichen Strukturen natür- 
lich zu kurz. Entsprechende Fragen definieren wichtige Themenstellungen der Mehrebenendiskussion (Huinink 1986).

Diskutiert man also die Bedeutung von Merkmalen der Herkunftsfamilie bzw. des Bildungsniveaus der Befragten oder unterschiedlicher gesellschaftlicher Bedingungen unterschiedlicher Kohorten in unterschiedlichen Altersklassen, so sind immer sowohl die situationsprägenden als auch die in den individuellen Entscheidungsproze $B$ und den Prozeß der Objektivierung individueller Handlungen intervenierenden Effekte zu berücksichtigen ${ }^{16}$.

Empirisch werden nur Beziehungen entlang der gestrichelten Pfeile im dargestellten Diagramm belegt werden. Der hier schon stark vereinfacht modellierte Prozeßzusammenhang muß dadurch nicht eindeutig repräsentiert sein, er kann sich über eine Reihe unterschiedlicher ,Einzelpfade“"vermitteln.

Gleichwohl kann eine theoretische Analyse im Rahmen dieses Modells präzise Hypothesen über die durch die gestrichelten Pfeile dargestellten Beziehungen liefern.

\subsection{Inhaltlich-theoretische Überlegungen}

Wenden wir uns nun zunächst der Frage zu, inwieweit den Bedingungen des Aufwachsens in der meist elterlichen Familie eine präformierende Relevanz für den individuellen Einstieg in die eigene Elternschaft zukommt.

Grob könnte man zunächst Indikatoren des sozialen Status der Herkunftsfamilie, wie die berufliche Stellung des Vaters und das Bildungsniveau der Eltern, als Hinweise auf die relative ökonomische Stellung, im Sinne der differentiellen, ökonomischen Ressourcenausstattung verstehen, die natürlich über das Spektrum individueller Orientierungen und Handlungsspielräume der Familienmitglieder mitentscheidet. Mit der Restriktivität der ökonomischen Ressourcen nimmt der Spielraum

if Darüber hinaus, und das wird ım Rahmen der Konstruktion solcher individualistisch angelegten Modellansätze häufig in zu geringem Maße gewürdigt. stellt die gesellschaftliche Bedingungsstruktur individueller Akteure auch ein wesentliches Bestimmungsmoment der Relevanz ihrer Aktionen im Prozeß der Veränderung dieser Bedingungen selbst dar. Ein solches Modell kommt in seiner elaborierten Version daher nie ohne einen Rückbezug auf die Makrostrukturen aus, der über ihren reinen Bedingungscharakter individueller Entscheidungsparameter hinausgeht. bei der Vermittlung von Lebensverlaufsperspektiven der Kinder $a b$. Insbesondere für Frauen lassen sich bei begrenzten Ressourcen die individuellen Voraussetzungen für eine längere Phase unabhängiger Lebensführung, z. B. eine qualifizierte berufliche Ausbildung, nur sehr viel schwieriger einklagen.

Neben der rein ökonomischen Dimension indizieren Merkmale des sozialen Status aber gerade auch soziale und gesellschaftliche Verortungsstrukturen einer Familie. Mit dem Status ist eine spezifische, differentielle Kompetenz und Anforderungsstruktur an gesellschaftliche Instanzen und Institutionen verknüpft. Dazu gehört auch eine bestimmte Stellung im ökonomischen Prozeß, also zum Beispiel auf dem Arbeitsmarkt. In diesem Kontext ist bei statusniedrigeren Familien eine engere Affinität zu eher traditionellen Normenmustern zu erwarten, in denen die Orientierung auf traditionelle Familienstrukturen mit ihren Formen der Arbeitsteilung eine zentrale Stellung einnimmt. Eine besondere Bedeutung kommt dabei der Einbettung der Familien in ihren sozialen Netzwerken oder auch Milieus zu. Erst über die Eingebundenheit in solchen als relativ homogen anzunehmenden Beziehungszusammenhängen vermittelt sich die gesellschaftliche Verankerung einzelner Personen und Familien. Insgesamt ist es somit plausibel, anzunehmen, daß der soziale Status der Herkunftsfamilie trotz möglicher intragenerationaler, sozialer Mobilität immer ein relevanter Faktor für die Disposition zu einer eigenen Familie sein dürfte. Das Aufwachsen in sozialen Kontexten, in denen die Akzeptanz früher Mutterschaft (niedriger sozialer Status) zum Beispiel ungebrochen bleibt, mit spezifischen Strukturen der partnerschaftlichen Suchprozesse, der Einstellung zur Sexualität und Familienplanung, der Auseinandersetzung mit gesellschaftlichen Prozessen und institutionellen Gegebenheiten, ergänzt die familialen Prozesse und hat somit einen Anteil an der „Präformierung“ individueller Lebensverläufe.

Mit dem sozialen Status der Herkunftsfamilie verbessert sich die informationelle Basis der Vermittlung alternativer Lebensperspektiven. Eltern mit einem höheren sozialen Status werden zu einem größeren Ausmaß Angebote individueller Optionen auf Lebensverwirklichung vermitteln. Dazu gehören ökonomische Unabhängigkeit, für Frauen damit eigene und gleichberechtigte Erwerbstätigkeit, und ein hoher Lebensstandard, der sich vor allem in den Ansprüchen an das Wohnen und die Freizeitgestaltung dokumentiert. Ihre Relevanz 
wird verstärkt und begründet ein Aspirationsniveau, dem ein früher Eintritt in die Elternschaft gerade unter den gegebenen gesellschaftlichen $\mathrm{Be}-$ dingungen als starke Einschränkung des Spielraums der individuellen Möglichkeiten entgegensteht. Auch zwischen den Kohorten dürfte sich der Widerspruch vergrößert haben und zu einer zunehmenden Benachteiligung größerer Familien geführt haben ${ }^{17}$.

Die Gründung einer eigenen Familie bot insbesondere den statusniedrigeren Mitgliedern der betrachteten Kohorten eine akzeptierte Möglichkeit, die elterliche Familie zu verlassen. Wollte man frühzeitig vom Elternhaus unabhängig sein - auf Gründe dafür wird noch eingegangen - blieb häufig nur die eigene Elternschaft. Vorliegende Ergebnisse weisen zwar nicht unbedingt auf einen positiven Zusammenhang des Alters beim Auszug aus dem Elternhaus und dem sozialen Status der Herkunftsfamilie hin. Bei den Frauen dürfte er aber eindeutig sein (Mayer/Wagner 1985; Ott 1985; Rindfuss/John 1983; Courgeau et al. 1986).

Die individuellen Erfahrungen im familialen Kontext lassen sich durch eine Reihe weiterer, für unsere Fragestellung relevanter Faktoren charakterisieren. Dazu gehören u. a. das Alter der Mutter bei der Geburt ihres ersten Kindes, die Zahl der Geschwister, das heißt auch die Größe der Herkunftsfamilie, die Tatsache, ob die befragte Person während ihrer Kindheit mit nur einem Elternteil aufgewachsen ist und die Bedeutung der Religion in der elterlichen Familie.

Das Alter der Mutter bei der Geburt des ersten Kindes kann die Vorstellungen zum Alter bei der Geburt des ersten Kindes der Befragten mitbestimmen. Hier würde also eine Übertragung bestimmter Wert- und Lebensorientierungen von einer auf die nachfolgende Generation erfolgen (Rindfuss/John 1983). So mag ein hohes Alter der Mutter bei der Geburt ihres ersten Kindes durch eine längere Erwerbstätigkeit begründet sein. Es ist anzunehmen, daß gerade für Frauen eine damit zu unterstellende stärkere Erwerbsorientierung der Mutter für die eigenen Lebenspläne an Relevanz gewinnt.

Auf der anderen Seite kann der größere Altersunterschied von Mutter und Kindern eine konfliktreichere Beziehung bedeuten. Diese wiederum könn-

${ }^{17}$ Eine ausführliche Analyse der dahinter stehenden gesellschaftlichen Entwicklung und der Motoren dieses Wandels erfolgt in Huinink 1987 a. te zu einem distanzierten Verhältnis zur eigenen Kindheit und möglicherweise eigener Elternschaft beitragen.

Mit der Größe der Herkunftsfamilie wird der für die einzelnen Kinder aufwendbare Ressourcenanteil geringer werden ${ }^{18}$. Wenn es aber zu Verteilungsproblemen der Ressourcen innerhalb von $\mathrm{Fa}$ milien kommt, darf man davon ausgehen, daß Mädchen systematisch benachteiligt werden. Zunächst wird versucht, den Jungen eine adäquate Ausbildung zu ermöglichen. Für die Mädchen wird mitunter eine frühzeitige Mutterkarriere als problemlose, alternative Perspektive akzeptiert. Mädchen werden auch in weit stringenterer Weise in den häuslichen Arbeitsprozeß einbezogen und gerade in großen Familien so zusätzlich auf eine eigene Hausfrauenkarriere ,vorbereitet“, so daß ein frühzeitiger Zeitpunkt der Geburt eigener Kinder wahrscheinlicher wird. Diese Argumentation legt nahe, daß ein empirischer Effekt nur bei den Frauen identifizierbar ist.

Gerade Kinder aus großen Familien dürften zudem versuchen, frühzeitiger den elterlichen Haushalt zu verlassen, sie sind möglicherweise auch aus ökonomischen Gründen dazu gezwungen (Rindfuss/John 1983; Courgeau et al. 1986). Ein weiteres Argument ist, daß in großen Familien die elterliche Kontrolle über die einzelnen Kinder zwangsläufig vermindert sein muß. Daher ist auch die Wahrscheinlichkeit zu ungewollten Schwangerschaften größer (Rindfuss/John 1983).

Geht man schließlich davon aus, daß das Aufwachsen mit vielen Geschwistern eine positive Bedeutung für die Beziehung zu Kindern und die Disposition zu eigenen Kindern hat. so muß man annehmen, daß Kinder aus kinderreichen Familien auch zu einem geringeren Umfang kinderlos bleiben und für den Fall der Vermeidung einer frühen Elternschaft später in größerem Umfang Geburten nachholen.

Eine Reihe von schon genannten Gründen sprechen dafür, daß auch Kinder, insbesondere Mädchen aus Ein-Eltern-Familien eher in eine eigene Familienkarriere eintreten. Auch sie versuchen, frühzeitiger die Familie zu verlassen, oder werden aufgrund der meist ökonomisch problematischen Lage dazu angehalten sein. Ein weiterer, oben schon zitierter Grund könnte auch hier die ge-

${ }^{18}$ Siehe unter diesem Gesichtspunkt die Diskussion zur Easterlin-Hypothese (Easterlin 1973, Oppenheimer 1976). 
ringere Kontrollmöglichkeit des einzelnen Elternteils über das jugendliche Kind sein (Michael/ Tuma 1985), allerdings weniger wegen der Anzahl der Kinder, als aufgrund der Tatsache, daß der alleinstehende Elternteil in der Regel vollerwerbstätig sein muß und die Kinder selbständiger aufwachsen.

Es ist aber zu fragen, ob die Erfahrung des Aufwachsens in einer Ein-Eltern-Familie, soweit sie tatsächlich schwierige ökonomische und insbesondere psychische Verhältnisse mit sich bringt, nicht die Orientierung auf eine eigene Familie in der Konkurrenz zu anderen Optionen und eigener Unabhängigkeit zurücktreten läßt. Familie als Lebenszusammenhang, der einen emotionalen Nutzen stiftet, steht eher außerhalb des Bewertungsspektrums $^{19}$.

Die These ist daher, daß Befragte, die in EinEltern-Familien aufgewachsen sind, eher schon in einem frühen Alter ihr erstes Kind bekommen, daß sie insgesamt aber zu einem höheren Anteil kinderlos bleiben.

Auf Indikatoren der religiösen Orientierung in der Herkunftsfamilie gehe ich hier nicht ausführlicher ein. Ihre Bedeutung dürfte gering sein bzw. zunehmend verschwinden. ${ }^{20}$.

Zur Charakterisierung des Bedingungskontextes, in dem die Befragten aufgewachsen sind, gehört auch der Wohnort der Herkunftsfamilie. Die Ausbildungsmöglichkeiten auf dem Land sind beschränkt, Zugangschancen zu Informationen und Erfahrungen über Alternativen zu eher traditionellen Lebensverläufen sind spärlicher. Das gilt zumindest noch weitgehend für die hier betrachteten Kohorten.

Die reine Exıstenz großstädtischer Opportunitäten reicht auf der anderen Seite nicht aus. Sie müssen auch erreichbar sein. Das heißt insbesondere bezogen auf Frauen, daß der Zugang positiv sanktioniert sein muß. In der Zeit, in der die betrachteten Kohorten aufgewachsen sind, dürfte das nicht durchgängig gelten. Obschon sich während der fünfziger Jahre die ökonomischen Bedingungen in der Bundesrepublik Deutschland ausgehend von den Großstädten erheblich verbessert haben, be-

${ }^{19}$ Siehe zur Argumentation speziell bezogen auf Männer auch Münkel 1984.

20 Dennoch können sie als "Schiene" für den Transport traditioneller Orientierungen begriffen und diskutiert werden. Ergebnisse bei den Männern scheinen das zu rechtfertigen. deutet dieses keineswegs, daß prinzipiell auch die Mädchen bzw. die Frauen direkt davon profitieren konnten. Ein Effekt ist daher erst bei den Frauen der jüngsten Kohorte zu erwarten.

In den bisherigen theoretischen und empirischen Analysen hat das Bildungsniveau bzw. das Niveau der beruflichen Ausbildung insbesondere bezogen auf Frauen eine besondere Beachtung gefunden ${ }^{21}$. Es kann als gesichert gelten, daB mit dem Niveau des Bildungsabschlusses die Zahl der Kinder abnimmt.

Dieses Ergebnis ist auch in unterschiedlicher Weise theoretisch abgeleitet worden. Zum Beispiel im Rahmen ökonomisch orientierter Ansätze läßt sich auf den Ansatz der Humankapitaltheorie verweisen (Zimmermann 1985). Danach ist zu erwarten, $\mathrm{da} B$ aufgrund eines höheren Bildungs- bzw. Ausbildungsabschlusses die Orientierung auf eine Erwerbstätigkeit zunimmt und eine frühzeitige Elternschaft in den Hintergrund drängt ${ }^{22}$.

Eher soziologisch orientierte Ansätze haben auf Fragen der Bedeutung der Bildungsbeteiligung für individuelle Orientierungen und Präferenzstrukturen zur Lebensplanung sowie für die sozialen Bedingungen des Lebensverlaufs einzugehen. Die Bildung beeinflußt die individuelle Definition der eigenen Geschlechterrolle und hat Auswirkungen auf die rollenspezifische Positionierung und gesellschaftliche Anerkennung (Freedman 1975; Scanzoni 1975).

Direkt anknüpfend an die Überlegungen zur Bedeutung der sozialen Herkunft läßt sich argumentieren, daß mit der Bildung das Anspruchsniveau für die zukünftige, eigene Lebensgestaltung zunimmt (Weiss 1986). Das gilt auch bezogen auf die potentiellen, eigenen Kinder und auf die Antizipation insbesondere des ökonomischen Aufwandes, der für sie erbracht werden muß. Eine frühzeitige Elternschaft würde der Befriedigung der präzise definierten Aspirationen entgegen stehen. Die Familiengründung wird kaum noch als Ausdruck der gesellschaftlichen Anerkennung gebraucht.

Besonders für die Männer bietet ein höheres Bildungsniveau aber auch eine größere Chance für

21 Es läßt sich natürlich belegen, daß das Bildungsniveau selbst deutlich mit Merkmalen der Herkunftsfamilie, wie sie diskutiert worden sind, zusammenhängen. Das ist bei der theoretischen Diskussion zu berücksichtigen.

${ }^{22}$ Siehe zum Beispiel auch die Argumentation bei Weiss 1986. 
eine ökonomische Absicherung, die in einem höheren Ausmaß die Ansprüche an eine eigene Familie realisierbar erscheinen läßt und ihnen gleichsam auch erst die ,Familienwürdigkeit" garantiert.

Die Ausgangsthese ist also, daß erstens ein höherer Bildungsabschluß zu einem späteren Zeitpunkt des Eintrittes in die Elternschaft führt, der nicht allein oder in erster Linie durch die unterschiedliche Dauer der Bildungs- bzw. Ausbildungsbeteiligung bedingt ist. Zweitens ist zu erwarten, daß der Anteil kinderloser Frauen und Männer mit dem Bildungsniveau zwar abnimmt, aber innerhalb der Bildungsgruppen bei den Frauen, in stärkerem Maße aber bei den Männern, solche mit einer beruflichen Ausbildung höhere Geburtenhäufigkeiten aufweisen dürften.

Es ist jedoch zu erwarten, daß sich der Bildungseffekt von Kohorte zu Kohorte verändert hat, wenn man sich die spezifischen historischen Situationen der Kohorten vor Augen hält. Da ist das Aufwachsen in der Nachkriegszeit mit äußerst schwierigen Verhältnissen, die unabhängig von der sozialstrukturellen Gruppe zu erheblichen Benachteiligungen in den sozialen und ökonomischen Realisierungschancen individueller Lebenspläne und insbesondere auch zu einem Aufschub der Geburt eigener Kinder führen mußten (Kohorte 1929-31).

Es folgt die Zeit des deutschen „Wirtschaftswunders", einer Zeit der ökonomischen wie sozialen "Restauration", in der der Eintritt in die Phase eigenständiger Lebensführung oft nur über eine eigene Familie zu verwirklichen war, was gesellschaftlich von der etablierten eher traditionellen Orientierung her gratifiziert wurde. Der Prozeß der zunehmenden sexuellen Enttabuisierung gewann an Bedeutung, gleichzeitig wurde mit der zunächst nur die höheren Altersgruppen erreichenden Diffusion sicherer Kontrazeptiva die gesellschaftliche Selbstverständlichkeit kompetenter und individuell fast sicher steuerbarer Familienplanung vorbereitet (Kohorte 1939-41).

Schließlich folgt die Zeit des größten Nachkriegsbooms und gesellschaftlichen Wandels, in der insbesondere jetzt auch für Frauen eine selbständigere Planung des eigenen Lebens außerhalb der traditionellen Muster möglich und ökonomisch erforderlich wird (Ausbildungsexpansion und Zunahme der Erwerbstätigkeit verheirateter Frauen), und sich insgesamt neue Standards in der Familienplanung, individueller Lebensführung und -gestaltung entwickeln und durchzusetzen beginnen (Kohorte $1949-51)^{23}$.
Die Hypothese zur Bedeutung des Bildungsniveaus für die Familienbildung läßt sich bezogen auf kohortenspezifische Veränderungen erweitern:

1. Die Veränderung der Altersstruktur bei der ersten Geburt ist mit zunehmendem Bildungsniveau bzw. bei abgeschlossener Berufsausbildung vergleichsweise geringer. Mit dem Bildungs- und insbesondere dem Ausbildungsniveau wächst das individuelle Kontrollpotential. Personen mit niedrigeren Bildungsabschlüssen bzw. ohne Ausbildung werden sehr viel stärker von gesellschaftlichen Kontrollstrukturen betroffen sein lind danach ihre Strategien auszurichten haben. Sie bekommen von Kohorte zu Kohorte zunehmend früher ihre ersten Kinder.

Sie trügen damit vornehmlich den Anstieg der aggregierten Geburtenhäufigkeiten in den jüngeren Altersstufen (siehe Graphik 1). Der erneute Anstieg der aggregierten Geburtenhäufigkeiten bei den älteren Frauen in der Kohorte 1949-51 müßte dagegen auf einen Kompositionseffekt zurückgeführt werden, der durch die Ausbildungsexpansion bei den Mädchen in den sechziger Jahren begründet wäre.

Bei den Männern können bildungsbedingte Chancendefizite eher eine Verzögerung der Familiengründung bedingen.

2. Das Ausmaß der Kinderlosigkeit dürfte mit dem Bildungsniveau von Kohorte zu Kohorte ansteigen. Die Orientierung auf und Einlösbarkeit von alternativen Lebensoptionen, wie sie in unserem Zusammenhang gerade für Frauen von Kohorte zu Kohorte zunehmen, ist hoch. Dazu gehören gesichertere Chancen zur Erwerbstätigkeit, die Erosion traditioneller Muster des Zusammenlebens. die von den höheren Bildungsgruppen ausgeht, und deren Motorfunktion bei der Neuformulicrung gesellschaftlicher Standards. Hinzu kommt, daß aufgrund der längeren Bildungsbeteiligung mit dem Bildungsniveau ein realisierter Aufschub durchaus gewünschter Geburten die Wahrscheinlichkeit, Altersschwellen zu überschreiten, jenseits derer eine eigene Elternschaft für problematisch erachtet wird, ansteigt. Auch diese These gilt für die Männer nur bedingt .

${ }^{23}$ Weitergehende Ergebnisse und Überlegungen sind im Rahmen des Lebensverlaufsprojekts schon vorgelegt worden. Siehe zum Beispiel Bloßfeld 1986, 1985a; Tölke 1986. Zur Bedeutung und Struktur dieses Wandels siehe auch Huinink $1987 \mathrm{a}$. 


\section{Methoden und Untersuchungsdesign}

\subsection{Das Analysemodell}

Die im folgenden erläuterten empirischen Ergebnisse wurden auf der Basis von Daten gewonnen, die in einer repräsentativen, retrospektiv angelegten Befragung der drei Geburtsjahrgangskohorten 1929-31, 1939-41 und 1949-51 im Rahmen des von K. U. Mayer geleiteten Projekts „Lebensverläufe und Wohlfahrtsentwicklung" des Sonderforschungsbereichs 3 der DFG (Mayer 1979, 1984) erhoben wurden. Die Erhebung fand zwischen Oktober 1981 und Mai 1983 statt $^{24}$. Mit ihr wurden umfassende Informationen zu den Lebensverläufen von 2171 Frauen und Männern der genannten Kohorten gewonnen, also Angaben zum Elternhaus, zur Bildungs-, Ausbildungs- und Erwerbskarriere, Migrationsgeschichte, zum Partnerschaftsverlauf inklusive der Heiratsgeschichte sowie zur Familienentwicklung.

Als Ziel war formuliert worden, Effekte der sozialen Herkunft und des Bildungsniveaus auf das Alter bei der Geburt des ersten Kindes zu identifizieren. Im Rahmen der Ereignisdatenanalyse bieten sich dazu Modelle mit altersklassenspezifisch konstanten Übergangsraten des Eintritts in die Elternschaft an (Huinink 1987b). Es werden keine globalen parametrischen Annahmen über den altersspezifischen Verlauf der Raten gemacht. Das betrachtete Altersintervall wird in Teilintervalle untergliedert, für die simultan je ein Intervallspezifisches Ratenmodell geschätzt wird.

Geht man von festen Altersintervallen aus, hier $18-22,22-26$ und $26-30$, und verzichtet man auf Informationen zu den höheren Altersklassen, insbesondere bei den älteren Kohorten, so daß keine durch den Interviewzeitpunkt zensierten Fälle zu berücksichtigen sind, kann man zu einer altersintervallspezifischen Schätzung der Übergangswahrscheinlichkeiten zum ersten Kind übergehen. Übergangsrate und Übergangswahrscheinlichkeit korrespondieren direkt miteinander (Rindfuss et al. 1984).

Daher werden hier Ergebnisse der Schätzungen von bedingten Übergangswahrscheinlichkeiten in den einzelnen Altersintervallen vorgestellt. Die Frage lautet also zum Beispiel: wie groß ist die

*4 Zum Erhebungsdesign siehe Brückner et al. 1984. Analysen zur Repräsentativität der Daten finden sich bei BloBfeld 1985 b.
Wahrscheinlichkeit, daß eine Frau oder ein Mann, die (der) bis zum Alter 22 noch kein erstes Kind bekommen hat, bis zum Alter 26 erstmalig Mutter (Vater) geworden ist?

\subsection{Die Variablen}

$\mathrm{Zu}$ den einbezogenen Variablen gehört ein dichotomisierter Indikator, der besagt, ob der Vater Angestellter, Beamter, Selbständiger (nicht Landwirte) bzw. Angehöriger einer Profession ist oder Arbeiter bzw. Landwirt (DVSTAT). Angaben zum Bildungsniveau der Eltern, die hoch mit anderen Merkmalen korreliert sind, werden nur in einer deskriptiven Einzelbetrachtung berücksichtigt. Die folgenden Variablen informieren über die Struktur der Herkunftsfamilie: die Anzahl der Geschwister (NGESCH), ein dichotomer Indikator, der angibt, ob die Befragten im Alter 15 in einer Ein-Eltern-Familie lebten (EINELT), und das Alter der Mutter der Befragten bei der Geburt des ersten Kindes (AMUTK1). Weiterhin ist zu berücksichtigen, ob der oder die Befragte katholisch ist (DCATH), und ob der oder die Befragte in einer Großstadt (Einwohnerzahl > 100000) gelebt hat (DGROSS).

Schließlich wird das Bildungs- bzw. Ausbildungsniveau kombiniert in einem Faktor operationalisiert. Die einzelnen Ausprägungen sind: Hauptschule ohne abgeschlossene Berufsausbildung (und darunter). Hauptschule mit abgeschlossener Berufsausbildung, mittlere Reife ohne Berufsausbildung, mittlere Reife mit Berufsausbildung, Abitur (DED1 bis DED5).

Die Variablen DKOH40 und DKOH50 geben an, ob die Befragten aus der Kohorte 1939-41 bzw. 1949-51 stammen. Bei den Männern unterbleibt die Differenzierung der Absolventen der mittleren Reife, da nahezu alle eine Berufsausbildung haben. Statt DKATH wird als Indikator die Rolle der Religion im elterlichen Haushalt (ROLREL) betrachtet, die auf einer Skala von 1 bis 10 gemessen wurde. Er erweist sich hier als erklärungskräftiger.

Als Übersicht zu den einbezogenen Merkmalen, ihren Mittelwerten und Varianzen, differenziert nach der Kohorte, dient die Tabelle 2. Sie informiert auch über Besetzungszahlen der einzelnen Teilgruppen in den Kohorten. 
Tabelle 2 Mittelwerte der Variablen in den Analysemodellen nach Geschlecht und Kohorte.

\begin{tabular}{|c|c|c|c|c|c|}
\hline & \multirow[b]{2}{*}{ Merkmal } & \multicolumn{4}{|c|}{ Kohorte } \\
\hline & & 1929-31 & $1939-41$ & $1949-51$ & gesamt \\
\hline \multirow[t]{15}{*}{ Frauen } & DVSTAT & 0.297 & 0.356 & 0.417 & 0.358 \\
\hline & NGESCH & 2.700 & 2.323 & 2.162 & 2.390 \\
\hline & EINELT & 0.087 & 0.225 & 0.067 & 0.125 \\
\hline & AMUTK 1 & 25.076 & 25.204 & 24.773 & 25.012 \\
\hline & DKATH & 0.486 & 0.440 & 0.473 & 0.467 \\
\hline & DGROSS & 0.150 & 0.249 & 0.249 & 0.217 \\
\hline & DED1 & 0.601 & 0.377 & 0.230 & 0.398 \\
\hline & DED2 & 0.201 & 0.344 & 0.426 & 0.326 \\
\hline & DED3 & 0.060 & 0.084 & 0.050 & 0.064 \\
\hline & DED 4 & 0.081 & 0.111 & 0.162 & 0.119 \\
\hline & DED5 & 0.057 & 0.084 & 0.132 & 0.092 \\
\hline & DKOH30 & & & & 0.324 \\
\hline & DKOH40 & & & & 0.327 \\
\hline & DKOH50 & & & & 0.349 \\
\hline & Anzahl der Fälle & 333 & 334 & 357 & 1024 \\
\hline \multirow[t]{14}{*}{ Männer } & DVSTAT & 0.319 & 0.339 & 0.419 & 0.360 \\
\hline & NGESCH & 2.652 & 2.274 & 2.011 & 2.295 \\
\hline & EINELT & 0.108 & 0.179 & 0.074 & 0.120 \\
\hline & AMUTK 1 & 25.343 & 25.774 & 25.586 & 25.574 \\
\hline & ROLREL & 5.929 & 5.681 & 5.182 & 5.588 \\
\hline & DGROSS & 0.180 & 0.265 & 0.291 & 0.247 \\
\hline & DED 1 & 0.294 & 0.165 & 0.068 & 0.173 \\
\hline & DED 2 & 0.505 & 0.615 & 0.516 & 0.546 \\
\hline & DED3 & 0.118 & 0.131 & 0.194 & 0.148 \\
\hline & DED 4 & 0.084 & 0.088 & 0.222 & 0.133 \\
\hline & $\mathrm{DKOH} 30$ & & & & 0.316 \\
\hline & $\mathrm{DKOH} 40$ & & & & 0.343 \\
\hline & DKOH50 & & & & 0.341 \\
\hline & Anzahl der Fälle & 326 & 353 & 351 & 1030 \\
\hline
\end{tabular}

\section{Ergebnisse der empirischen Analyse}

Ich stelle nun die Ergebnisse der Schätzung der Logit-Modelle für die Wahrscheinlichkeiten einer ersten Geburt innerhalb der drei Altersklassen $18-22,22-26,26$ bis 30 sowie bis zum Alter 30 überhaupt vor. In die Schätzung für eine Altersklasse gehen jeweils nur die Individuen ein, die zu Beginn des Altersintervalls noch kinderlos waren.

In der Tabelle 3 sind die Ergebnisse der Modellschätzungen zu den oben angegebenen Altersintervallen für Frauen und Männer aller drei Kohorten unter der Einbeziehung der eingeführten Herkunfts- und Bildungsvariablen dokumentiert .

Variablen mit positiven (negativen) Koeffizienten in einer Altersklasse kovariieren dabei positiv (ne- gativ) mit den geschätzten bedingten Wahrscheinlichkeiten der Geburt eines ersten Kindes in der jeweiligen Altersklasse ${ }^{25}$.

Es lassen sich eine Reihe charakteristischer Unterschiede der Effekte einzelner Merkmale in den verschiedenen Altersklassen erkennen, die durchweg den Ableitungen aus den theoretischen Überlegungen entsprechen. So gilt bei den Frauen:

${ }^{25}$ Für die Schätzung der individuellen Übergangswahrscheinlichkeiten im Altersintervall a, $p_{1}^{A}$ gilt: $p_{1}^{A}=\exp$ $\left(x_{1} \cdot \beta^{a}\right) /\left(1+\exp \left(x_{1} \cdot \beta^{a}\right)\right)$, wobei $x_{1}$ der Vektor der Ausprägungen der einbezogenen Variablen für das Individuum und $\beta^{a}$ der Vektor der Koeffizienten für das Altersintervall a ist. 
Tabelle 3 Ergebnisse des Logit-Modells der bedingten Übergangswahrscheinlichkeiten zum ersten Kind für die Altersklassen 18-22, 22-26, 26-30 und unter 30, Frauen und Männer.

\begin{tabular}{|c|c|c|c|c|}
\hline \multirow{2}{*}{$\begin{array}{l}\text { Frauen } \\
\text { Merkmal }\end{array}$} & \multicolumn{4}{|c|}{ Altersklasse } \\
\hline & $18-20$ & $22-26$ & $26-30$ & -30 \\
\hline DVSTAT & $-0.50250^{\star \star \star}$ & $-0.26308^{\star}$ & -0.17986 & $-0.40414^{\star \star}$ \\
\hline NGESCH & $0.05698^{*}$ & -0.00272 & 0.07340 & $0.06410^{*}$ \\
\hline EINELT & 0.22674 & -0.03122 & $-0.66528^{\star \star}$ & -0.32414 \\
\hline AMUTK1 & $-0.03496^{\star \star}$ & -0.00854 & $0.04552^{\star}$ & 0.00732 \\
\hline DKATH & -0.12212 & 0.06626 & -0.12868 & -0.02870 \\
\hline DGROSS & -0.24700 & -0.24502 & $-0.45320^{\star}$ & $-0.40398^{* *}$ \\
\hline \multicolumn{2}{|l|}{ DED 1} & \multicolumn{3}{|c|}{ Referenzkategorie } \\
\hline DED2 & $-0.33962^{\star}$ & 0.13404 & $0.74678^{\star \star \star}$ & $0.35432^{\star}$ \\
\hline DED3 & -0.01028 & $-0.51996^{*}$ & -0.28678 & -0.42522 \\
\hline DED 4 & $-1.17468^{\star \star \star}$ & -0.37098 & 0.11598 & $-0.49392^{*}$ \\
\hline DED 5 & $-2.45326^{\star \star \star}$ & $-1.08872^{\star \star \star}$ & -0.34646 & $-1.32460^{\star \star * *}$ \\
\hline \multicolumn{2}{|l|}{ DKOH30 } & \multicolumn{3}{|c|}{ Referenzkategorie } \\
\hline DKOH 40 & $0.40556^{\star \star}$ & $0.78928^{\star \star \star}$ & -0.04576 & $0.53556^{\star \star \star}$ \\
\hline DKOH50 & $1.11198^{\star \star \star}$ & -0.18154 & -0.36514 & 0.04160 \\
\hline Konstante & -0.16698 & -0.12380 & -1.17478 & 1.17152 \\
\hline \multicolumn{2}{|l|}{ Männer } & \multicolumn{3}{|l|}{ Altersklasse } \\
\hline Merkmal & $18-20$ & $22-26$ & $26-30$ & -30 \\
\hline DVSTAT & 0.01458 & $-0.33728^{*}$ & 0.08752 & -0.12136 \\
\hline NGESCH & -0.01340 & 0.00596 & -0.02256 & -0.00748 \\
\hline ROLREL & -0.05282 & 0.04034 & $0.11880^{\star * *}$ & $0.09058^{\star \star \star}$ \\
\hline DGROSS & -0.03322 & -0.25902 & -0.21716 & $-0.26040^{\star}$ \\
\hline EINELT & 0.33684 & $-0.39792^{*}$ & -0.06562 & -0.17636 \\
\hline AMUTK 1 & -0.02206 & $-0.04068^{* *}$ & -0.02294 & $-0.03768^{\star \star \star}$ \\
\hline \multicolumn{2}{|l|}{ DED 1} & \multicolumn{3}{|c|}{ Referenzkategorie } \\
\hline DED2 & -0.08862 & $0.30750^{\star}$ & $0.49948^{\star \star}$ & $0.49074^{\star \star \star}$ \\
\hline DED3 & $-0.72094^{*}$ & $-0.67898^{\star *}$ & $0.65404^{* *}$ & 0.13800 \\
\hline DED 4 & $-1.75110^{\star \star \star}$ & $-1.09098^{\star \star \star}$ & 0.02852 & $-0.50788^{\star}$ \\
\hline \multicolumn{2}{|l|}{ DKOH 30} & \multicolumn{3}{|c|}{ Referenzkategorie } \\
\hline DKOH 40 & 0.16668 & $0.28614^{\star}$ & $0.40780^{* *}$ & $0.45444^{* * *}$ \\
\hline DKOH 50 & $0.47460^{*}$ & $-0.29140^{\star}$ & -0.27294 & $-0.27916^{\star}$ \\
\hline Konstante & -1.52638 & 0.06508 & -0.79820 & 0.78478 \\
\hline
\end{tabular}

${ }^{* * *}$ : sig. z. N. 0.01; $\quad$ **: sig. z. N. 0.05; $\quad$ *: sig. z. N: 0.15

- Das Alter der Mutter der Befragten bei der Geburt des ersten Kindes korreliert deutlich mit dem Alter der Befragten selbst bei der Geburt ihres ersten Kindes: sein Effekt auf die bedingte Wahrscheinlichkeit für die Geburt eines ersten Kindes ist in der Altersklasse 18-22 noch negativ, in der Altersklasse 26-30 ist er dagegen positiv.

- Frauen, die im Alter 15 in der Großstadt gelebt haben, haben durchgängig geringere Übergangswahrscheinlichkeiten zum ersten Kind: es resultiert ein Niveaueffekt bis zum Alter 30.

- Frauen, deren Vater einen höheren beruflichen Status hat bzw. kein Landwirt ist, bekommen deut- lich seltener im Alter unter 26 ihr erstes Kind und bleiben bis zum Alter 30 zum größeren Teil kinderlos.

- Das Aufwachsen in Ein-Eltern-Familien begünstigt offenbar ein früheres Alter bei der Geburt des ersten Kindes. Deutlicher ist aber der negative Effekt der Variablen in dem höchsten Altersintervall. So bestätigt sich die These, nach der für Kinder aus Ein-Eltern-Familien einem erhöhten Risiko früher Schwangerschaft ein höherer Anteil an Kinderlosigkeit gegenübersteht.

- Die Zahl der Geschwister ist nichtlinear mit dem Alter bei der Geburt des ersten Kindes ver- 
knüpft. Positive Effekte zeigen sich in der unteren und der oberen Altersgruppe. Damit ist also auch die größere Disposition von Frauen aus kinderreichen Familien zu eigenen Kindern in späteren Altersphasen belegt, nachdem sie zunächst aus verschiedenen Gründen eine frühe Schwangerschaft vermieden haben.

- Die Effekte des Bildungs- bzw. Ausbildungsniveaus werden sehr deutlich: bei den Hauptschülerinnen mit Ausbildung werden die Geburten stark in die höhere Altersgruppe verschoben, aber bis zum Alter 30 gegenüber den Hauptschülerinnen ohne Ausbildung nachgeholt. Auch die Realschulabsolventinnen mit beruflicher Ausbildung und die Abiturientinnen bekommen ihr Kind in einem höheren Alter, bleiben aber auch zu einem zunehmenden Anteil kinderlos.

Besonders ist festzuhalten, daß die Merkmale der Herkunftsfamilie auch unter Kontrolle des Bildungsabschlusses deutliche Effekte behalten. Es läßt sich zeigen, daß sie natürlich ohne die Einbeziehung der Bildungsvariablen deutlich höher sind; auch die Bildungsniveaus der Eltern werden dann wieder statistisch interessant. Der Bildungseffekt dürfte also zum Teil nur intermediär sein.
Nur wenige Bemerkungen zu den Ergebnissen bei den Männern. Befragte, deren Mutter bei der Geburt ihres ersten Kindes schon älter war, bleiben zu einem größeren Anteil kindellos. Die Rolle der Religion im elterlichen Haushalt kovariiert mit einem späteren Eintritt in die Elternschaft, aber auch mit einem höheren Niveau der Geburtenhäufigkeit bis zum Alter 30, wie die positiven Effekte für das Altersintervall 26-30 und bis 30 zeigen. Die Verzögerungseffekte des Bildungsniveaus sind kleiner als bei den Frauen. Die Hauptschüler ohne Ausbildung beginnen erst später bzw. weniger früher als die höheren Bildungsgruppen eine Familienkarriere. Diese Differenzierung war bei der theoretischen Diskussion des Bildungseffekts schon vermutet worden. Damit korrespondiert, daß der positive Effekt der Ausbildung bei den Hauptschülern größer ist als bei den Frauen.

Die vorgestellten Ergebnisse können zu irreführenden Verallgemeinerungen beitragen, da Kohorteneffekte bislang nur additiv berücksichtigt worden sind, aber begründeterweise kohortenspezifische Differenzierungen der Effekte einzelner Merkmale angenommen werden müssen. Ich bin im theoretischen Teil an verschiedenen Stellen schon darauf eingegangen.

Tabelle 4 Anteile kinderloser Frauen in den Kohorten 1929-31, 1939-41 und 1949-51 im Alter 22, 26, 30 und 40 nach unterschiedlichen sozialstrukturellen Merkmalen.

\begin{tabular}{|c|c|c|c|c|c|c|c|c|c|c|c|c|}
\hline \multirow[t]{3}{*}{ Merkmal } & \multirow[t]{3}{*}{ Auspr. } & \multicolumn{11}{|c|}{ Kohorte und Alter } \\
\hline & & \multicolumn{4}{|c|}{$1929-31$} & \multicolumn{4}{|c|}{$1939-41$} & \multicolumn{3}{|c|}{$1949-51$} \\
\hline & & 22 & 26 & 30 & 40 & 22 & 26 & 30 & 40 & 22 & 26 & 30 \\
\hline DVSTAT & $\begin{array}{l}0 \\
1\end{array}$ & $\begin{array}{l}73.8+ \\
85.9\end{array}$ & $\begin{array}{l}42.5+ \\
63.6\end{array}$ & $\begin{array}{l}19.3+ \\
34.3\end{array}$ & $\begin{array}{r}9.9 \\
10.1\end{array}$ & $\begin{array}{l}65.3+ \\
84.9\end{array}$ & $\begin{array}{l}26.9+ \\
46.2\end{array}$ & $\begin{array}{c}15.3+ \\
23.5\end{array}$ & $\begin{array}{r}8.8 \\
13.4\end{array}$ & $\begin{array}{l}53.4+ \\
76.5\end{array}$ & $\begin{array}{l}35.6+ \\
57.7\end{array}$ & $\begin{array}{l}18.3+ \\
38.9\end{array}$ \\
\hline DMBILD & $\begin{array}{l}0 \\
1\end{array}$ & $\begin{array}{l}76.7 \\
83.9\end{array}$ & $\begin{array}{l}48.8 \\
48.4\end{array}$ & $\begin{array}{l}23.3 \\
29.0\end{array}$ & $\begin{array}{r}9.6 \\
12.9\end{array}$ & $\begin{array}{l}70.2+ \\
87.5\end{array}$ & $\begin{array}{l}29.8+ \\
62.5\end{array}$ & $\begin{array}{l}15.9+ \\
35.0\end{array}$ & $\begin{array}{l}8.8+ \\
22.5\end{array}$ & $\begin{array}{l}59.8+ \\
87.8\end{array}$ & $\begin{array}{l}42.1+ \\
65.9\end{array}$ & $\begin{array}{l}23.4+ \\
53.7\end{array}$ \\
\hline DVBILD & $\begin{array}{l}0 \\
1\end{array}$ & $\begin{array}{l}75.2+ \\
92.9\end{array}$ & $\begin{array}{l}46.6+ \\
64.3\end{array}$ & $\begin{array}{l}22.8 \\
31.0\end{array}$ & $\begin{array}{r}9.7 \\
11.9\end{array}$ & $\begin{array}{l}70.0+ \\
82.8\end{array}$ & $\begin{array}{l}29.6+ \\
53.4\end{array}$ & $\begin{array}{l}15.9+ \\
29.3\end{array}$ & $\begin{array}{r}9.4 \\
15.5\end{array}$ & $\begin{array}{l}58.0+ \\
84.1\end{array}$ & $\begin{array}{l}39.9+ \\
65.2\end{array}$ & $\begin{array}{l}22.9+ \\
43.5\end{array}$ \\
\hline NGESCH & $\begin{array}{l}\leqslant 2 \\
>2\end{array}$ & $\begin{array}{l}75.8 \\
79.9\end{array}$ & $\begin{array}{l}46.0 \\
53.0\end{array}$ & $\begin{array}{l}23.7 \\
23.9\end{array}$ & $\begin{array}{r}11.1 \\
8.2\end{array}$ & $\begin{array}{l}76.2+ \\
65.6\end{array}$ & $\begin{array}{l}37.6+ \\
27.2\end{array}$ & $\begin{array}{l}20.5 \\
14.4\end{array}$ & $\begin{array}{r}11.4 \\
8.8\end{array}$ & $\begin{array}{l}69.6+ \\
49.6\end{array}$ & $\begin{array}{l}49.2+ \\
35.9\end{array}$ & $\begin{array}{l}30.0+ \\
20.5\end{array}$ \\
\hline EINELT & $\begin{array}{l}0 \\
1\end{array}$ & $\begin{array}{l}77.9 \\
72.4\end{array}$ & $\begin{array}{l}50.2 \\
34.5\end{array}$ & $\begin{array}{l}23.8 \\
24.1\end{array}$ & $\begin{array}{r}9.6 \\
13.8\end{array}$ & $\begin{array}{l}72.7 \\
70.7\end{array}$ & $\begin{array}{l}33.1 \\
36.0\end{array}$ & $\begin{array}{l}16.9 \\
22.7\end{array}$ & $\begin{array}{l}10.0 \\
12.0\end{array}$ & $\begin{array}{l}63.7 \\
54.2\end{array}$ & $\begin{array}{l}44.7 \\
45.8\end{array}$ & $\begin{array}{l}26.1 \\
37.5\end{array}$ \\
\hline DGROSS & $\begin{array}{l}0 \\
1\end{array}$ & $\begin{array}{l}77.0 \\
80.0\end{array}$ & $\begin{array}{l}48.6 \\
50.0\end{array}$ & $\begin{array}{l}22.3 \\
32.0\end{array}$ & $\begin{array}{r}8.9 \\
16.0\end{array}$ & $\begin{array}{l}71.4 \\
74.7\end{array}$ & $\begin{array}{l}31.3 \\
41.0\end{array}$ & $\begin{array}{l}15.1+ \\
27.7\end{array}$ & $\begin{array}{c}7.5+ \\
19.3\end{array}$ & $\begin{array}{l}59.7+ \\
73.0\end{array}$ & $\begin{array}{l}40.7+ \\
57.3\end{array}$ & $\begin{array}{l}25.0 \\
32.6\end{array}$ \\
\hline ED & $\begin{array}{l}1 \\
2 \\
3 \\
4 \\
5\end{array}$ & $\begin{array}{l}73.5+ \\
77.3 \\
75.0 \\
96.3 \\
94.7\end{array}$ & $\begin{array}{l}45.0+ \\
42.4 \\
60.0 \\
74.1 \\
63.2\end{array}$ & $\begin{array}{l}22.0 \\
18.2 \\
30.0 \\
37.0 \\
36.8\end{array}$ & $\begin{array}{r}10.5 \\
6.1 \\
15.0 \\
7.4 \\
15.8\end{array}$ & $\begin{array}{l}61.1+ \\
73.3 \\
75.0 \\
91.9 \\
89.3\end{array}$ & $\begin{array}{l}22.2+ \\
31.0 \\
39.3 \\
48.6 \\
71.4\end{array}$ & $\begin{array}{l}13.5+ \\
12.9 \\
25.0 \\
27.0 \\
42.9\end{array}$ & $\begin{array}{r}9.5 \\
7.8 \\
21.4 \\
10.8 \\
14.4\end{array}$ & $\begin{array}{l}40.2+ \\
59.9 \\
55.6 \\
75.9 \\
99.9\end{array}$ & $\begin{array}{l}26.8+ \\
36.8 \\
44.4 \\
55.2 \\
89.4\end{array}$ & $\begin{array}{l}15.9+ \\
15.8 \\
38.9 \\
34.5 \\
68.1\end{array}$ \\
\hline DKATH & $\begin{array}{l}0 \\
1\end{array}$ & $\begin{array}{l}80.0 \\
74.7\end{array}$ & $\begin{array}{l}48.8 \\
48.8\end{array}$ & $\begin{array}{l}24.1 \\
23.5\end{array}$ & $\begin{array}{r}9.4 \\
10.5\end{array}$ & $\begin{array}{l}68.1+ \\
77.6\end{array}$ & $\begin{array}{l}34.0 \\
33.3\end{array}$ & $\begin{array}{l}18.1 \\
18.4\end{array}$ & $\begin{array}{l}10.6 \\
10.2\end{array}$ & $\begin{array}{l}64.9 \\
60.9\end{array}$ & $\begin{array}{l}47.9 \\
41.4\end{array}$ & $\begin{array}{l}28.7 \\
24.9\end{array}$ \\
\hline
\end{tabular}


Mit Ausnahme des Bildungsniveaus diskutiere ich die Kohortenunterschiede nur für die Frauen zunächst an Hand der deskriptiven Darstellungen der Tabelle $4^{26}$. Sie enthält analog zur Tabelle 1 altersspezifische Anteile kinderloser Frauen, hier aber differenziert nach der Zugehörigkeit zu den diskutierten sozialstruktuellen Teilgruppen. Zusätzlich wird die Bildung der Mutter (DMBILD) bzw. des Vaters (DVBILD) berücksichtigt. Die dichotomen Variablen geben an, ob die Eltern mindestens einen Realschulabschluß absolviert haben.

Es zeigt sich, daß der insgesamt identifizierte, negative Effekt von DGROSS kohortenspezifisch zu differenzieren ist. Während er in den älteren Kohorten nur in der Altersklasse 30-40 auftritt, wird er in der jüngsten Kohorte schon in den jüngeren Altersklassen bedeutsam: die Differenz der Anteile kinderloser Frauen ist hier schon beim Alter 22 signifikant. Wenn man in Betracht zieht, daß die Altersklasseneinteilung recht grob ist, kann man von einer Diffusion des Effekts in die jüngeren Altersstufen sprechen. Das entspricht genau der These, daß für die Frauen erst im Laufe der sechziger Jahre der "Großstadtvorteil" in Anspruch genommen werden konnte und die Kohorten sukzessive in jüngerem Alter davon profitierten.

Der im Alter nichtlineare Effekt der Anzahl der Geschwister tritt erst in der letzten Kohorte auf ${ }^{27}$. Insbesondere fallen die geringen Unterschiede der Anteile kinderloser Frauen mit zwei und weniger vs. mehr als zwei Geschwistern in der älteren Kohorte auf.

Die Effekte des „Syndroms“ sozialer Status der Herkunftsfamilie/Bildung gewinnen von Kohorte zu Kohorte an Bedeutung ${ }^{28}$. Ich schließe die Herkunftsfamilie hier ausdrücklich mit ein. Die Kohorte 1929-31 war noch von den Folgen der Nachkriegszeit betroffen. In der jüngsten Kohorte dage-

${ }^{26}$ Die Aussagen zu den in Tabelle 4 dokumentierten bivariaten Ergebnissen sind durch kohortenspezifische Schätzungen der multivariaten Logit-Schätzungen, die ich aus Platzgründen nicht ausführlicher darstelle, für die einzelnen Kohorten abgesichert. Die Besetzungszahlen in den einzelnen Kategorien sind mit der Ausnahme bei der Geschwisterzahl aus der Tabelle 2 ableitbar.

${ }^{27}$ Das wird in dem entsprechenden Logit-Modell für die Kohorte 1949-51 deutlich belegt.

28 Scheller (1986) kommt zu einer ähnlichen Aussage Die eigenen Logit-Modelle für die einzelnen Altersintervalle weisen bei der ältesten Kohorte auch die geringste Diskriminierungskraft dieser Merkmale aus. gen entstehen die Wurzeln der gesellschaftlichen Neuorientierung ab dem Ende der sechziger Jahre. Sie weist daher eine große, systematische Heterogenität auf, die auf vorübergehende Polarisierungstendenzen im Geburtenverhalten hindeutet.

Abschließend soll nun die Frage empirisch beantwortet werden, inwieweit tatsächlich die theoretisch postulierte, differentielle Sensibilität unterschiedlicher Bildungsgruppen auf die unterschiedlichen zeithistorischen Bedingungen gegeben ist. Die Veränderung der Altersstruktur der Geburtenhäufigkeiten von Kohorte zu Kohorte (siehe Graphik 1) läßt sich im Prinzip durch eine Kombination von Effekten der Verhaltensänderung in bildungshomogenen Teilpopulationen und der veränderten sozialstrukturellen Komposition nach diesen Teilpopulationen erklären. Daß letzteres eindeutig eine Rolle gespielt hat, läßt sich in der Tabelle 2 aus dem rapiden Anstieg des Anteils von Frauen mit einer abgeschlossenen Berufsausbildung ableiten. Ein Teil der im Aggregat festzustellenden „Rückverlagerung" der Geburten in höhere Altersstufen in der Kohorte 1949-51 ließe sich durch diesen Anstieg ausgebildeter Frauen begründen (Kompositionseffekt), wenn sich der Anteil der Befragten aus dieser Bildungsgruppe, die ein vergleichsweise hohes Alter bei der ersten Geburt aufweisen, nicht entscheidend verändert hat ${ }^{29}$. Dagegenzusetzen wäre der starke Zuwachs von jungen Müttern bei den Hauptschülerinnen ohne Berufsausbildung, deren Anteil an der Gesamtpopulation aber von Kohorte zu Kohorte klar abnimmt.

Einen genaueren Einblick geben die bildungsspezifischen Kohorteneffekte bei den Frauen, die ich als Ergebnisse eines Logit-Modells gewinne, das aus dem oben vorgestellten durch die zusätzliche Einbeziehung der Interaktion zwischen dem Bildungsabschluß und der Kohortenzugehörigkeit der Befragten hervorgeht. In der Graphik 2 sind die Bildungsgruppen-bezogenen Koeffizienten der Kohortenvariablen in dem erweiterten Logit-Modell für jede der vier Altersgruppen gegen die jeweilige Kohorte abgetragen worden. Für die Referenzkohorte 1929-31 sind sie sämtlich gleich 0 gesetzt.

In der Altersgruppe 18-22 nimmt der Kohorteneffekt in der niedrigsten Bildungsgruppe insbesonde-

\footnotetext{
${ }^{29}$ Siehe zur Frage der Dekomposition von Kompositions- und Verhaltenseffekten bezogen auf die EheschlieBung auch Papastefanou 1986 b.
} 

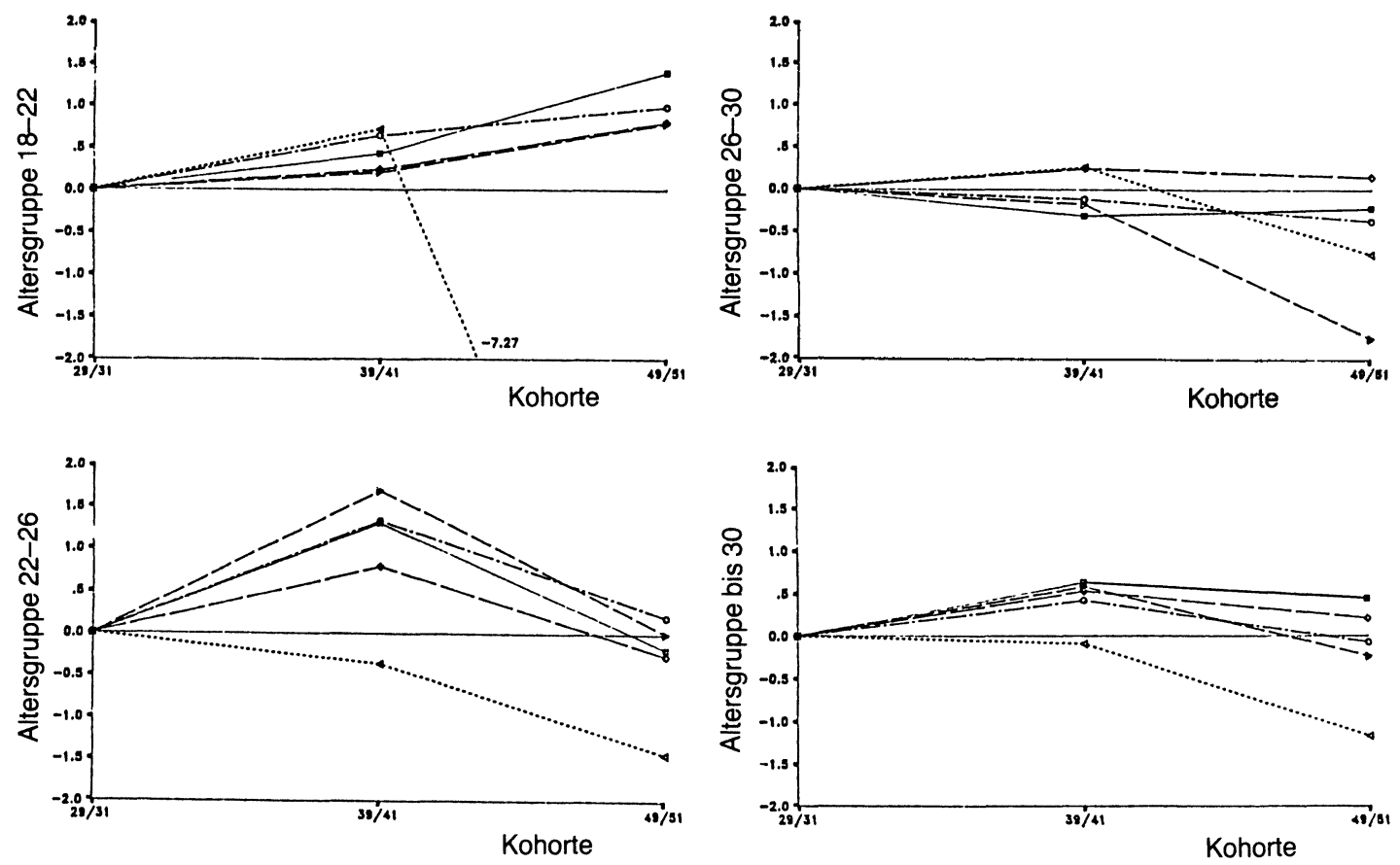

Graphik 2 Bildungsgruppenspezifische Kohorteneffekte auf die Übergangswahrscheinlichkeit zum ersten Kind in den Altersgruppen 18-22, 22-26, 26-30 und bis 30; Frauen.

Die Bildungsgruppen sind:

Hauptschule ohne Ausbildung

und darunter

Hauptschule mit Ausbildung

re von der Kohorte 1939-41 zur jüngsten Kohorte erheblich zu. Die Zuwächse sind signifikant. Bei den mittleren Bildungsabschlüssen ist der Anstieg schwächer und nicht mehr signifikant. Für die Abiturientinnen, die nur sehr geringe Geburtenhäufigkeiten in diesem Altersintervall aufweisen, zeigen sich starke, wohl erratische Schwankungen der Effekte.

Im Altersintervall von 22-26 steigt bei der niedrigsten Bildungsgruppe der Effekt in der mittleren Kohorte signifikant an. Der erneute Rückgang bei der Kohorte 1949-51 auf das Niveau der ältesten Kohorte steht in direkter Beziehung zu der weiteren, starken Vorverlagerung des Alters (siehe Altersgruppe 18-22). Die mittleren Bildungsgruppen weisen eine ähnliche Struktur der kohortenspezifischen Effekte auf, aber entweder abgeschwächt (Hauptschülerinnen mit Ausbildung) und in keinem Fall signifikant oder ohne Entsprechung in der jüngsten Altersklasse, wie sie bei den Hauptschülerinnen ohne Ausbildung festgestellt wurde.
Mittlere Reife ohne Ausbildung

Mittlere Reife mit Ausbildung

Abitur

Bei den Abiturientinnen nehmen die Effekte von Kohorte zu Kohorte deutlich und statistisch signifikant ab.

In der Altersklasse 26-30 verringern sich die Koeffizienten von Kohorte zu Kohorte nur schwach. Korrespondierend zur Entwicklung in den jüngeren Altersgruppen liegen sie für die Kohorten 1939-41 bzw. 1949-51 bei den Hauptschülerinnen ohne Ausbildung leicht unterhalb, bei den Hauptschülerinnen mit Ausbildung leicht oberhalb des Niveaus der Kohorte 1929-31. Graphik 2 zeigt schließlich auch, daß das Ausmaß der Kinderlosigkeit bis zum Alter 30 nur in der Gruppe mit einem höheren formalen Bildungsniveau, insbesondere mit Abitur, von Kohorte zu Kohorte zunimmt.

Die theoretischen Ableitungen zur Bedeutung der Beziehung der Zugehörigkeit zu einer bestimmten Bildungsgruppe und einer der drei Kohorten für die Altersstruktur bei der ersten Geburt sind damit weitgehend bestätigt. 


\section{Kurzes Resümee}

In diesem Beitrag sind eine Reihe theoretischer Überlegungen zur Beziehung von sozialer Herkunft und Bildung zum Alter beim ersten Kind entwickelt worden. Ihnen konnten empirische Einzelergebnisse gegenübergestellt werden, die aus der Schätzung von Modellen bedingter Wahrscheinlichkeiten der Geburt erster Kinder in unterschiedlichen Altersintervallen für ausgewählte Kohorten gewonnen wurden.

Sie belegen die Vermutung, da $\beta$ den Bedingungen des Aufwachsens in der elterlichen Familie eine erhebliche Bedeutung für die individuellen Familienbildungsprozesse zukommt, auch wenn man das erreichte Bildungsniveau kontrolliert. Überraschend ist vielleicht die deutliche, direkte Relevanz von Indikatoren des sozialen Status der Eltern. So wird die These bestätigt, daß gerade in solch sensiblen Bereichen des individuellen Lebenslaufs, wie sie die eigene Elternschaft darstellt, Erfahrungen in der eigenen Kindheit mit ihren sozialen und ökonomischen Bedingungen und dort erfahrene Routinen, eine große Bedeutung behalten. Es ist zu vermuten, daß dies um so mehr gilt, als die Geburt von Kindern nie allein die Folge rationaler Entscheidungsprozesse, in denen die Vor- und Nachteile kühl abgewogen werden, sein dürfte.

Die Ergebnisse geben auch einen Einblick in die soziologische Struktur der demographischen Entwicklung. wie sie zu Anfang skizziert wurde. Wir haben gesehen, daß diese Entwicklung stark durch Veränderungen der Altersstruktur bei der Geburt eines Kindes, speziell des ersten Kindes bedingt waren. Die Vorverlagerung des Alters im Aggregat reflektiert eine verhaltensbedingte Veränderung in den eher niedrigeren sozialen Schichten, deren Anteil über die Kohorten hin aber abnimmt. Der starke Rückgang der Erstgeburten Ende der sechziger/Anfang der siebziger Jahre wird in beträchtlichem Maße durch eine Umschichtung in der sozialstrukturellen Zusammensetzung der Kohorten eingeleitet, die insbesondere durch den starken Anstieg des Anteils von Frauen mit Berufsausbildung in den jüngeren Kohorten gekennzeichnet ist. Im Gegensatz zu den nichtausgebildeten Frauen bleibt der Anteil der Frauen mit Berufsausbildung, die erst später ihr erstes Kind bekommen, relativ groß. Das drückt sich in den steigenden aggregierten Geburtenhäufigkeiten nach dem Alter 25 aus.

Die Bildungsexpansion bei den allgemeinbildenden Abschlüssen hat die betrachteten Generatio- nen noch nicht erreicht, auch wenn die Anteile höherer Abschlüsse erheblich ansteigen. Doch die enorme Vergrößerung der Zahl kinderloser Frauen und Männer in der letzten Kohorte, zumindest bis zum Alter 30, läßt erahnen, welchen Anteil auch die nach 1970 stark zunehmende Partizipation an höherer Ausbildung an dem Prozeß der beherrschenden Verbreitung eines späten Eintritts in familiale Karriere gehabt haben wird.

\section{Literatur}

Birg, H., Huinink, J., Koch, H., Vorholt, H., 1984: Kohortenanalytische Darstellung der Geburtenentwicklung in der Bundesrepublik Deutschland. IBSMaterialien Nr. 10, Bielefeld.

Bloom, D. E., Trussel. J., 1984: What are the Determinants of Delayed Childbearing and Permanent Childlessness in the United States? Demography 21: 591-611.

BloBfeld, H.-P., 1985 a: Berufseintritt und Berufsverlauf. Eine Kohortenanalyse über die Bedeutung des ersten Berufs in der Erwerbsbiographie. Mitteilungen aus der Arbeitsmarkt- und Berufsforschung 18: 177-197.

Bloßfeld, H.-P., 1985b: Zur Repräsentativität der Sfb-3Lebensverlaufsstudie. Ein Vergleich mit Daten aus der amtlichen Statistik. Arbeitspapier Nr. 163 des Sfb 3. Frankfurt und Mannheim.

Bloßfeld, H.-P., 1986: Bildungsverläufe im historischen Wandel. Eine Längsschnittanalyse über die Veränderung der Bildungsbeteiligung im Lebenslauf dreier Geburtskohorten, Arbeitspapier 225 des Sfb 3. Frankfurt und Mannheim.

Boudon, R., 1983: Individual Action and Social Change: A No-Theory of Social Change. The British Journal of Sociology 34: 1-18.

Brückner, E., Hoffmeyer-Zlotnik, J., Kirschner, H.-P., Wiedenbeck, M., 1984: Methodenbericht ,Lebensverläufe'. Zuma-Technischer Bericht Nr. T 84/08. Mannheim.

Courgeau, D., Leliévre, E., Wagner, M., 1986: Leaving Home and Marriage in France and Germany. Manuskript, Berlin und Paris.

Dinkel, R., 1983: Analyse und Prognose der Fruchtbarkeit am Beispiel der Bundesrepublik Deutschland. Zeitschrift für Bevölkerungswissenschaft 9: 47-72.

Easterlin, R. A., 1973: Relative Economic Status and the American Fertility Swing. In: Sheldon, E. B. (Hrsg.), Family Economic Behavior: Problems and Prospects, Philadelphia: Lippincott.

Freedman, R., 1975: The Sociology of Human Fertility: An Annotated Bibliography. New York: Irvington Publishers.

Freedman, R., 1979: Theories of Fertility Decline: A Reappraisal. Social Forces 58: 1-17.

Heinsohn, G., Knieper, R., Steiger, O., 1979: Menschenproduktion - Allgemeine Bevölkerungstheorie der Neuzeit. Frankfurt: Suhrkamp. 
Hoffmann-Nowotny, H. J., Höpflinger, F., Kühne, F., Ryffel, C., Erni, D., 1985: Planspiel Familie. Diessenhofen: Rüegger

Höhn, Ch., 1982: Der Familienzyklus - Zur Notwendigkeit einer Konzepterweiterung. Boppard: Boldt.

Höhn, Ch., 1986: Einflußfaktoren des generativen Verhaltens. Zeitschrift für Bevölkerungsforschung 12: 309-323.

Huinink, J., 1986: Mehrebenensystem-Modelle in den Sozialwissenschaften. Dissertation. Bielefeld.

Huinink, J., 1987a: Kohortenanalyse der Geburtenentwicklung in der Bundesrepublik Deutschland. Erscheint in: Herlth, A., Strohmeier, K. P. (Hrsg.), Familie und Lebenslauf, Leverkusen: Leske + Budrich.

Huinink, J., 1987b: Soziale Herkunft, Bildung und Eintritt in die Elternschaft. Erste Ergebnisse im Vergleich dreier Geburtsjahrgangskohorten. Arbeitspapier Nr. 229 des Sfb 3. Frankfurt und Mannheim.

Kiefl, W., Schmid, J., 1985: Empirische Studien zum generativen Verhalten. Boppard: Boldt.

Linde, H.. 1984: Theorie der säkularen Nachwuchsbeschränkung 1800 bis 2000. Frankfurt: Campus.

Mackensen, R., 1985: Bevölkerungswissenschaft im Umbau. Zeitschrift für Bevölkerungswissenschaft 11: 193-218.

Marini, M. M., 1978: The Transition to Adulthood: Sex Differences in Educational Attainment and Age at Marriage. American Sociological Review 43: 483-507.

Marini, M. M., 1984: Women's Educational Attainment and the Timing of Entry into Parenthood. American Sociological Review 49: 491-511.

Marini, M. M., 1985: Determinants of the Timing of Adult Role Entry. Social Science Research 14: 309-350.

Marschalck, P., 1984: Bevölkerungsgeschichte Deutschlands im 19. und 20. Jahrhundert. Frankfurt: Suhrkamp.

Mayer, K. U., 1979: Lebensverläufe und Wohlfahrtsentwicklung. S. 181-217 in: Antrag auf Einrichtung und Finanzierung des Sfb 3 ..Mikroanalytische Grundlagen der Gesellschaftspolitik". Frankfurt und Mannheim.

Mayer, K. U., 1984: Lebensverläufe und Wohlfahrtsentwicklung. S. 119-142 in: Bericht über die Forschungstätigkeit in der zweiten Forschungsphase 1982-84. Frankfurt und Mannheim.

Mayer, K. U., Wagner, M., 1986: Der Auszug von Kindern aus dem elterlichen Haushalt. S, 1-42 in: Zimmermann, K. F. (Hrsg.), Demographische Probleme der Haushaltsökonomie. Bochum: Brockmeyer.

Michael, R. T.. Tuma, N. B., 1985: Entry into Marriage and Parenthood by Young Men and Women: The Influence of Family Background. Demography 22: 515-544.

Münkel, W., 1984: Geburtenrückgang als Folge veränderten generativen Handelns des Mannes. Zeitschrift für Bevölkerungswissenschaft 10: 193-207.
Newman, J. L., McCulloch, Ch. E., 1984: A Hazard Rate Approach to the Timing of Births. Econometrica 52: 939-961.

Oppenheimer, V. K., 1976: The Easterlin Hypothesis: Another Aspect of the Echo to Consider. Population and Development Review 2: 433-457.

Ott, N., 1985: Ausscheiden erwachsener Kinder aus dem elterlichen Haushalt. S. 43-79 in: Zimmermann, K. F. (Hrsg), Demographische Probleme der Haushaltsökonomie. Bochum: Brockmeyer.

Papastefanou, G., 1986a: Zur Bedeutung des Berufszugangs für die Geschlechts- und Kohortendifferenzierung des Heiratsprozesses. Manuskript. Berlin.

Papastefanou, G., 1986b: Die Veränderung des Heiratsprozesses durch die Bildungsexpansion in der Bundesrepublik Deutschland. Manuskript. Berlin.

Pohl, K., 1982: Konzeption und derzeitiger Stand der Paneluntersuchung des Bundesinstituts für Bevölkerungsforschung zu Fragen des Familienbildungsprozesses. Zeitschrift für Bevölkerungswissenschaft 8: 499-521.

Rindfuss, R. R., Bumpass, L., John, C. St., 1980: Education and Fertility: Implication for the Roles Women Occupy. American Sociological Review 45: 431-447.

Rindfuss, R. R., Morgan, Ph. S., Swicegood, L. G., 1984: The Transition to Motherhood: The Intersection of Structural and Temporal Dimensions. American Sociological Review 49: 359-372.

Rindfuss, R. R., John, C. St., 1983: Social Determinants of Age at First Birth. Journal of Marriage and the Family 45: 553-565.

Scanzoni, J., 1975: Sex Roles, Life Styles, and Childbearing. New York: Free Press.

Scheller, G., 1986: Erklärungsversuche des Wandels im Heirats- und Familiengründungsalter seit 1950. Zeitschrift für Bevölkerungswissenschaft 11: 549-576.

Schmid, J., 1984: Bevölkerung und soziale Entwicklung. Der demographische Übergang als soziologische und politische Konzeption. Boppard; Boldt.

Schubnell, H., 1972: Der Geburtenrückgang in der Bundesrepublik Deutschland. Stuttgart: Kohlhammer.

Tölke, A., 1986: Historische Ausgangssituation und Veränderung im Ausbildungs- und Erwerbsverhalten junger Frauen in der Nachkriegszeit. In: Voges. W. (Hrsg.), Methoden der Biographie- und Lebensverlaufsforschung. Leverkusen: Leske + Budrich.

Tuma. N. B., Hannan. M. T., 1984: Social Dynamics. Models and Methods. Orlando: Academic Press.

Weiss, Th., 1986: Ökonomische Bestimmungsgründe der Fertilität in westlichen Industrieländern. Materialien zur Bevölkerungswissenschaft. Sonderheft 5 . Wiesbaden.

Zimmermann, K.-F., 1985: Familienökonomie. BerlinHeidelberg-New York-Tokio: Springer. 